\title{
Frequency dependent thermal expansion in binary viscoelastic composites
}

\author{
James G. Berryman ${ }^{1, *}$ \\ ${ }^{1}$ University of California, Lawrence Berkeley National Laboratory, \\ 1 Cyclotron Road, MS 90R1116, Berkeley, CA 94720, USA
}

\begin{abstract}
The effective thermal expansion coefficient $\beta^{*}$ of a binary viscoelastic composite is shown to be frequency dependent even if the thermal expansion coefficients $\beta_{A}$ and $\beta_{B}$ of both constituents are themselves frequency independent. Exact calculations for binary viscoelastic systems show that $\beta^{*}$ is related to constituent values $\beta_{A}, \beta_{B}$, volume fractions, and bulk moduli $K_{A}, K_{B}$, as well as to the overall bulk modulus $K^{*}$ of the composite system. Then, $\beta^{*}$ is determined for isotropic systems by first bounding (or measuring) $K^{*}$ and therefore $\beta^{*}$. For anisotropic systems with hexagonal symmetry, the principal values of the thermal expansion $\beta_{\perp}^{*}$ and $\beta_{\|}^{*}$ can be determined exactly when the constituents form a layered system. In all the examples studied, it is shown explicitly that the eigenvectors of the thermoviscoelastic system possess non-negative dissipation - despite the complicated analytical behavior of the frequency dependent thermal expansivities themselves. Methods presented have a variety of applications from fluid-fluid mixtures to fluid-solid suspensions, and from fluid-saturated porous media to viscoelastic solid-solid composites.
\end{abstract}

Keywords: composites, thermal expansion, viscoelasticity

*JGBerryman@LBL . GOV 


\section{Introduction}

Viscoelastic relaxation dynamics can be important in many fields of science (Christensen, 1982; Lakes, 1999; Schmalholz et al., 1999; Angell et al., 2000; Hestholm et al., 2006). But the role played by thermal expansion in complex situations with multiple viscoelastic constituents present has seldom been addressed. Thermal expansion coefficients $\beta$ in homogeneous (pure) materials are normally treated as being real quantities (Biot, 1956; Fei, 1995), even in materials that are known to be viscoelastic. One goal of the present work is to emphasize the importance of considering frequency dependence of thermal expansion, especially in viscoelastic composite materials, because it is not difficult to show (as we shall see) that effective thermal expansion of composites will normally exhibit frequency dependence (inherited from the viscoelastic constants of the constituents) even if all the individual constituents have effectively frequency independent thermal expansion properties.

Frequency dependence of thermal expansion can come into play in two ways: First, a sudden change of temperature on the sample surface will take time to migrate into the interior. This migration process can have different rates, associated with thermal conductivities of the constituents in a composite medium. So the rate of thermal front (not necessarily a very sharp front since this is a diffusive process) propagation into the composite will be complicated. Then, in addition, the thermal expansion of each constituent interacts with the local stress-strain fields. The final state (i.e., at long times, assuming no further changes in temperature or external stress-strain state occur) will be achieved gradually over a period of time with larger initial oscillations giving way to smaller ones as some of the excess energy is lost due to viscoelastic dissipation. So it is natural (as we show here) that the thermal expansion of a viscoelastic composite should be frequency dependent - acquiring its frequency dependence from the viscoelastic constants, even if the individual constituents have truly frequency-independent thermal expansion properties.

A second way in which frequency dependent thermal expansion may occur happens when a viscoelastic composite is repeatedly stressed and released. Such cycling will produce viscous losses of energy to the system, causing it to heat locally at different rates since we are considering composite systems. Resulting thermal gradients will gradually smooth out as temperature becomes more uniform. But, we see that this process results in a similar response to that of suddenly imposed external temperature change. Again thermal expansion effects 
are nonuniform due to nonuniform local heating and will result in complicated interactions between thermal expansion and viscoelastic creep losses to heat.

For a viscoelastic system, there are at last two distinct and important sets of constants: one for zero frequency (very long times) and one for infinite freuqency (very short times) (Vinogradov and Milton, 2005; Hsieh and Tuan, 2006; 2007). Therefore, much of the analysis of viscoelastic systems can be concentrated on certain fixed frequencies (for example, $f=0$ and $f=\infty)$. So we will not dwell on the details of the frequency dependence of $\beta^{*}$ itself, or that of the usual viscoelastic constants here, but rather concentrate on establishing the complex nature of $\beta^{*}$ — thereby making the main point of the paper. Textbooks are available (Christensen, 1982; Lakes, 1999) on the frequency dependent behavior of the viscoelastic constants, and the time-dependent thermal expansion behavior then follows directly from the formulas discussed here.

Methods similar to the main one used to relate overall thermal expansion coefficients of composites to overall bulk modulus have also been used in other physical contexts by various authors (Cribb, 1968; Schulgasser, 1989; Berryman and Milton, 1991; 1992). In particular, the method has sometimes been called the "method of uniform fields" (Dvorak, 1990; Benveniste and Dvorak, 1990; 1992; Dvorak and Benveniste, 1992; 1997), although it is nevertheless basically the same idea used by Cribb (1968) in the thermoelastic media context. A similar approach applied in a much wider context has also been introduced by Grabovsky et al. (2000).

We consider binary viscoelastic composites, and elaborate two cases: (1) composites having overall statistically isotropic behavior, and (2) composites having hexagonal symmetry especially those having transversely isotropic symmetry due to layering of two constituents.

\section{Isotropic binary viscoelastic composites}

As illustrated in Figure 1, we first consider a binary composite containing two types of constituents labelled respectively $\mathrm{A}$ and $\mathrm{B}$. The shape of the constituent will play no role in the present section of the paper, but it will come into play in the next section because of assumed layering of the components. 


\subsection{Thermal expansion for viscoelastic systems}

If the volume strain is $\delta \epsilon$ and the entropy per unit volume is $\delta s$, then small changes in these quantities are related to small changes in pressure $\delta p$ and temperature $\delta \theta$ by the pertinent equations for a thermoelastic medium which are:

$$
\left(\begin{array}{c}
\delta \epsilon \\
\delta s
\end{array}\right)=\left(\begin{array}{cc}
\frac{1}{K} & 3 \beta \\
3 \beta & c_{p} / T
\end{array}\right)\left(\begin{array}{c}
-\delta p \\
\delta \theta
\end{array}\right),
$$

where $K$ is the (isothermal) bulk modulus, $3 \beta$ is the (volume) thermal expansion coefficient, $c_{p}$ is the heat capacity, $T$ is the ambient system temperature measured in ${ }^{\circ} K$, and $s=\rho \hat{S}$ where $\rho$ is the mass density and $\hat{S}$ is the entropy per unit mass (also known as the specific entropy). The related adiabatic bulk modulus (for $\delta s \equiv 0$ ) is $K_{a d}=K\left(1-9 \beta^{2} K T / c_{p}\right)^{-1}$ (Ferry, 1970). We emphasize smallness of the independent and dependent variables with the $\delta$ 's to limit the discussion to linear systems, in which case we do not need to consider the dependence of the coefficients, $K, \beta$, and $c_{p}$ on the applied fields. When the overall composite is isotropic and each of the constiuents is also isotropic, Eq. (1) applies separately to each constituent's behavior as well as to the overall behavior of the composite. We then add subscripts A and B for the two constituents' moduli, stresses ( $\delta p$ or $\delta \sigma)$ and strains $(\delta \epsilon)$, as necessary, and use $*$ superscripts to indicate the overall behavior. In particular, the (small) volume strain $\delta \epsilon=\delta \epsilon_{11}+\delta \epsilon_{22}+\delta \epsilon_{33}$ is the sum of the linear strains in the $x_{1}, x_{2}$, and $x_{3}$ directions, respectively. The isotropic pressure change is $\delta p$ and this is related to the changes in the principal stresses $\sigma_{11}$, etc., by $-\delta p=\left(\delta \sigma_{11}+\delta \sigma_{22}+\delta \sigma_{33}\right) / 3$. The change in temperature is $\delta \theta$. The change in entropy is $\delta s$, while $c_{p}$ is the specific heat per unit volume at constant pressure (Landau and Lifshitz, 1986).

Effective overall thermal expansion coefficient $\beta^{*}$ for an isotropic composite composed of isotropic constituents can be written as

$$
\beta^{*}=\langle\beta\rangle+\frac{\beta_{B}-\beta_{A}}{\frac{1}{K_{B}}-\frac{1}{K_{A}}}\left(\frac{1}{K^{*}}-\left\langle\frac{1}{K}\right\rangle\right) .
$$

If the volume fraction of the isotropic constituents are respectively $f_{A}$ and $f_{B}$, then

$$
\langle\beta\rangle \equiv f_{A} \beta_{A}+f_{B} \beta_{B}
$$

is the volume average of $\beta$ and

$$
\left\langle\frac{1}{K}\right\rangle \equiv \frac{f_{A}}{K_{A}}+\frac{f_{B}}{K_{B}}
$$


is the inverse of the Reuss average (i.e., the harmonic mean) of the constituent bulk moduli $K_{A}$ and $K_{B}$. The effective overall bulk modulus of the (assumed) isotropic composite is $K^{*}$. The elastic version of the result (2) is well-known (Levin, 1967; Cribb, 1968; Rosen and Hashin, 1970; Christensen, 1979), but the formula will be rederived later in this subsection for the viscoelastic case.

Also note that several other equivalent forms of (2) are available, the simplest of which is probably Levin's (1967):

$$
\frac{\beta^{*}-\beta_{A}}{\beta_{B}-\beta_{A}}=\frac{\frac{1}{K^{*}}-\frac{1}{K_{A}}}{\frac{1}{K_{B}}-\frac{1}{K_{A}}} .
$$

This form is not symmetrical in $A$ and $B$, whereas (2) is symmetrical. But there is a second form of (5) that is also true (as will next be shown) and it is easily obtained by interchanging the $A$ and $B$ subscripts. In fact, these two forms both follow (after some algebra) from (2), and vice versa. Again note that (5) depends on volume fractions only through the implicit dependence of the overall value on system bulk modulus $K^{*}$.

The derivation of these results for the viscoelastic (and therefore frequency dependent) case follows from (1) by first asking whether it is possible to find combinations of $\delta p$ and $\delta \theta$ such that $\delta p=\delta p_{A}=\delta p_{B}, \delta \theta=\delta \theta_{A}=\delta \theta_{B}$, while $\delta \epsilon=\delta \epsilon_{A}=\delta \epsilon_{B}$. If so, then

$$
\delta \epsilon=-\frac{\delta p}{K^{*}}+\beta^{*} \delta \theta=-\frac{\delta p_{A}}{K_{A}}+\beta_{A} \delta \theta_{A}=-\frac{\delta p_{B}}{K_{B}}+\beta_{B} \delta \theta_{B} .
$$

These relations imply that

$$
\beta^{*}-\frac{\delta p}{K^{*} \delta \theta}=\beta_{A}-\frac{\delta p_{A}}{K_{A} \delta \theta_{A}}=\beta_{B}-\frac{\delta p_{B}}{K_{B} \delta \theta_{B}},
$$

and since - by hypothesis - both fields are uniform, we also have $\delta p / \delta \theta=\delta p_{A} / \delta \theta_{A}=$ $\delta p_{B} / \delta \theta_{B}$. The implications that we can draw are therefore that

$$
\begin{aligned}
& \beta^{*}-\beta_{A}=\left(\frac{1}{K^{*}}-\frac{1}{K_{A}}\right) \frac{\delta p}{3 \delta \theta}, \\
& \beta^{*}-\beta_{B}=\left(\frac{1}{K^{*}}-\frac{1}{K_{B}}\right) \frac{\delta p}{3 \delta \theta},
\end{aligned}
$$

and most importantly the fact that this happens specifically for the ratio

$$
\frac{\delta p}{3 \delta \theta}=\frac{\beta_{B}-\beta_{A}}{1 / K_{B}-1 / K_{A}}=\frac{\delta p_{A}}{3 \delta \theta_{A}}=\frac{\delta p_{B}}{3 \delta \theta_{B}} .
$$

Substituting the last expression into the previous two gives both (5) and its interchange version (where $A \rightarrow B \rightarrow A$ ). Then averaging these two expressions with respect to volume 
fraction gives (2). Note that nothing in the derivation changes if we assume the moduli $K_{A}=$ $K_{A}(\omega)$ and/or $K_{B}=K_{B}(\omega)$, and $K^{*}=K^{*}(\omega)$, are all functions of angular frequency $\omega=$ $2 \pi f$. So the form (2) holds true at every frequency $\omega$, and $\beta^{*}=\beta^{*}(\omega)$ inherits its frequency dependence directly from $K_{A}(\omega)$ and $K_{B}(\omega)$ even if the constituent thermal expansions $\beta_{A}$ and $\beta_{B}$ are not themselves functions of frequency.

As this derivation shows, there is no restriction on the nature of the thermal expansion moduli, or the elastic(real)/viscoelastic(complex) moduli - time independent or time dependent processes. There are however some implicit restrictions in that the thought experiment itself assumes an overall uniform state, but is silent on how long it might take to achieve such a state in practice - thereby, implicitly assuming long times (and therefore low frequencies). Also, it is clear that the various moduli must not themselves be dependent on the magnitude of the applied stresses, or strains, or temperatures. So this requirement is one of the linearity of the equations, which for practical purposes means the applied physical changes must not be too large in magnitude or the problem will become a nonlinear one. There may still be some comparable statements possible under those high stress/temperature conditions, but they will need to be carefully applied to derivatives of the appropriate energy functionals assuming that such exist for the corresponding problems of interest. However, we will not pursue this extension of the methods here.

It is clear from (2) and (5) that $\beta^{*}$ for a viscoelastic composite is frequency dependent if either $K_{A}$ or $K_{B}$ is frequency dependent. It is known that $K^{*}$ will also be frequency dependent under these circumstances (Gibiansky and Milton, 1993), and exceedingly unlikely that frequency dependencies on the right hand side of equations (2) and (5) would exactly cancel out.

Another especially important point to consider is that, although microstructure does not enter the formulas (2) and (5) explicitly, the influence of the microstructure is nevertheless present because of the overall bulk modulus $K^{*}$ in both equations, and this bulk modulus itself can be a strong function of the microstructure.

\subsection{Bounds on bulk modulus $K^{*}$}

When the details of the microstructure of the binary composite are unknown but it is nevertheless known that an assumption of statistical isotropy is adequate, it is then ap- 
propriate to consider variational bounding methods (Gibiansky and Milton, 1993; Milton and Berryman, 1997) for the complex moduli. The work of Gibiansky and Milton (1993) is especially pertinent since the estimates we need are those on the complex bulk modulus $K^{*}$.

Defining the complex strain $\epsilon$, complex stress $\sigma$, and complex stiffness $C$ (which varies in space because of the presence of two distinct constituents), we have the viscoelastic constitutive relationship:

$$
\sigma=C \epsilon
$$

We can usefully think of this as a $6 \times 6$ system of equations: $\sigma$ and $\epsilon$ are each $1 \times 6$ complex vectors, and $C$ is a $6 \times 6$ matrix (using the Voigt convention for transforming elastic or viscoelastic tensors into matrices).

If real parts are distinguished by single primes and imaginary parts by double primes, then we can define real, composite vectors

$$
j=\left(\begin{array}{c}
\epsilon^{\prime \prime} \\
\sigma^{\prime \prime}
\end{array}\right) \quad \text { and } \quad e=\left(\begin{array}{c}
-\sigma^{\prime} \\
\epsilon^{\prime}
\end{array}\right),
$$

corresponding to the $12 \times 12$ system

$$
j=D e,
$$

where now

$$
D=\left(\begin{array}{cc}
\left(C^{\prime \prime}\right)^{-1} & \left(C^{\prime \prime}\right)^{-1} C^{\prime} \\
C^{\prime}\left(C^{\prime \prime}\right)^{-1} & C^{\prime \prime}+C^{\prime}\left(C^{\prime \prime}\right)^{-1} C^{\prime}
\end{array}\right)
$$

is a $12 \times 12$ real matrix.

There is still a lot of work to do to produce the bounds, but now we have transformed the problem so we can deal (for the moment) only with real quantities. We have to analyze the behavior of the average $D$ matrix for our binary composite, but this is relatively straightforward within the context of random media theory. We can for example generalize the Hashin-Shtrikman variational principle for this more complicated problem. The results are bounds on both complex bulk modulus (Gibiansky and Milton, 1993) and complex shear modulus (Milton and Berryman, 1997). The details are contained in the quoted references, so we will skip ahead to some pertinent developments concerning the quantities called the "canonical functions" and Milton's Y-tensor (Milton, 1987).

First, let us define a complex function $\Lambda(u)$, which will be called "the canonical function 
for bulk modulus." If the symbol \langle\rangle is the spatial averaging operator, then

$$
\Lambda(u) \equiv\left\langle\frac{1}{K(\vec{r})+\frac{4}{3} u}\right\rangle^{-1}-\frac{4}{3} u .
$$

The number $u$ can be real or complex, and has the significance in many cases of being a pertinent shear modulus for the system of interest. The number $u$ will always have the dimensions $(\mathrm{GPa})$ of a bulk or shear modulus. Another function of similar type can be defined for the shear modulus as

$$
\Gamma(y) \equiv\left\langle\frac{1}{G(\vec{r})+y}\right\rangle^{-1}-y .
$$

This function is called by analogy "the canonical function for shear modulus." When all the moduli are real, the Hashin-Shtrikman upper and lower bounds (Hashin and Shtrikman, 1963) on $K^{*}$ can be expressed in terms of $\Lambda(u)$ by setting $u=\operatorname{either} G_{A}$ or $G_{B}$. If $G_{\max }$ is the larger of the two, then $\Lambda\left(G_{\max }\right) \geq K^{*}$ is the upper bound on the real bulk modulus, while setting $u=G_{\min }$ produces the lower bound. Setting $u=0$ (or $y=0$ for $G$ ) gives the Reuss average (the harmonic mean), while taking $u \rightarrow \infty$ (or $y=\infty$ for $G$ ) produces the Voigt average (the arithmetic mean). The Hashin-Shtrikman-Walpole bounds (Walpole, 1969) for shear modulus are similarly expressed by defining the two $y$ values $y_{\max }=\left(G_{\max } / 6\right)\left(9 K_{\max }+\right.$ $\left.8 G_{\max }\right) /\left(K_{\max }+2 G_{\max }\right)$, and $y_{\min }=\left(G_{\min } / 6\right)\left(9 K_{\min }+8 G_{\min }\right) /\left(K_{\min }+2 G_{\min }\right)$, where $G_{\text {max }}=\operatorname{Max}\left(G_{A}, G_{B}\right), K_{\text {min }}=\operatorname{Min}\left(K_{A}, K_{B}\right)$, etc.

These facts about real $\Lambda$ (and also $\Gamma$ ) are not directly useful to us for the complex (viscoelastic) problem, but nevertheless they perhaps help to motivate the use of the complex version of the function for different purposes. In particular, for the viscoelastic problem, Gibiansky and Milton (1993) show that four values of $\Lambda(u)$ are important for the bounds on complex $K^{*}$ - and, furthermore, they are exactly the same ones we have just discussed although now generalized to the complex plane. In particular, $K_{1 *} \equiv \Lambda\left(G_{A}\right), K_{2 *} \equiv \Lambda\left(G_{B}\right)$, $K_{h *} \equiv \Lambda(0)=\left\langle\frac{1}{K(\vec{r})}\right\rangle^{-1}$, and $K_{a *}=\Lambda(\infty)=\langle K(\vec{r})\rangle=f_{A} K_{A}+f_{B} K_{B}$. The functional form is the same as that shown in (15), but now the constants appearing inside $\Lambda$ (i.e., $K(\vec{r})$ and $u$ ) are permitted to be complex numbers (but the volume fractions continue to have their usual real significance).

Gibiansky and Milton (1993) define four arcs in the complex $K$-plane, each arc passing through the first two points (i.e., $p=K_{1 *}, K_{2 *}$ ), and also through one of the remaining four 
points $(p)$ according to:

$$
\operatorname{Arc}\left(K_{1 *}, K_{2 *}, K_{h *}\right), \quad \operatorname{Arc}\left(K_{1 *}, K_{2 *}, K_{a *}\right), \quad \operatorname{Arc}\left(K_{1 *}, K_{2 *}, K_{A}\right), \quad \operatorname{Arc}\left(K_{1 *}, K_{2 *}, K_{B}\right)
$$

where

$$
\operatorname{Arc}\left(p_{1}, p_{2}, p_{3}\right) \equiv \phi_{1} p_{1}+\phi_{2} p_{2}-\frac{\phi_{1} \phi_{2}\left(p_{1}-p_{2}\right)^{2}}{\phi_{2} p_{1}+\phi_{1} p_{2}-p_{3}}
$$

and the parameter $\phi_{1}=1-\phi_{2}$ is real and varies along the interval $[0,1]$. The resulting function is a type well-known in complex analysis called a linear fractional, or bilinear, transformation.

These results can be usefully compared to the previously defined canonical functions and also to the related $Y$-tensor (or $Y$-transform) of Milton (1987) If the actual effective constants to be determined (or estimated using the bounding arguments) are $K^{*}$ and $G^{*}$ (bulk and shear moduli respectively), then we can define arguments $Y_{K}$ and $Y_{G}$ such that

$$
K^{*}=\Lambda\left(3 Y_{K} / 4\right) \quad \text { and } \quad G^{*}=\Gamma\left(Y_{G}\right)
$$

The canonical functions are one-to-one, and they are analytically invertible. So we find directly that

$$
Y_{K}=\frac{K_{A} K_{B}\left(K^{*}\langle 1 / K\rangle-1\right)}{\langle K\rangle-K^{*}}
$$

and

$$
Y_{G}=\frac{G_{A} G_{B}\left(G^{*}\langle 1 / G\rangle-1\right)}{\langle G\rangle-G^{*}} .
$$

Thus, we have turned the canonical functions inside out to find formulas for the two $Y$ 's. This process is useful because, if we can now obtain bounds directly on the $Y$ 's, then we obtain rather tight bounds on both $K^{*}$ and $G^{*}$. For example, in the case of real constants, the trivial bounds $0 \leq Y_{K}, Y_{G} \leq \infty$ obviously result in the Reuss (1929) and Voigt (1928) bounds for both moduli $K^{*}$ and $G^{*}$. The bounds $\frac{4 G_{\min }}{3} \leq Y_{K} \leq \frac{4 G_{\max }}{3}$ for $Y_{K}$ result in the Hashin-Shtrikman bounds on bulk modulus (Hashin and Strikman, 1963); and a similar statement holds for $G^{*}$ and $Y_{G}$, where the Hashin-Shtrikman-Walpole points (Walpole, 1969) become the bounds on $Y_{G}$ for real moduli.

For the viscoelastic problem, we have the general $Y$-tensor given by:

$$
Y\left(D^{*}, D_{A}, D_{B}\right)=\left(\begin{array}{cc}
\left(y^{\prime \prime}\right)^{-1} & -\left(y^{\prime \prime}\right)^{-1} y^{\prime} \\
-y^{\prime}\left(y^{\prime \prime}\right)^{-1} & y^{\prime \prime}+y^{\prime}\left(y^{\prime \prime}\right)^{-1} y^{\prime}
\end{array}\right)
$$


by analogy to (14). And the $6 \times 6$ matrix $y$ is defined by

$$
y\left(C^{*}, C_{A}, C_{B}\right)=-f_{B} C_{A}-f_{A} C_{B}+f_{A} f_{B}\left(C_{A}-C_{B}\right)\left(f_{A} C_{A}+f_{B} C_{B}-C^{*}\right)^{-1}\left(C_{A}-C_{B}\right) .
$$

For isotropic composites, this matrix can be split out easily into its bulk and shear parts.

For example, when this has been done for the shear modulus (Milton and Berryman, 1997), we can show (recalling that single primed objects are real parts, and double primed objects are imaginary parts) that:

$$
\left(\begin{array}{cc}
\frac{1}{2 y_{G}^{\prime \prime}} & -\frac{y_{G}^{\prime}}{y_{G}^{\prime \prime}} \\
-\frac{y_{G}^{\prime}}{y_{G}^{\prime}} & 2\left[y_{G}^{\prime \prime}+\frac{\left.y_{G}^{\prime}\right)^{2}}{y_{G}^{\prime \prime}}\right]
\end{array}\right)-Z \geq 0
$$

Here $Z$ is a $2 \times 2$ real comparison matrix. This statement says that the spectrum of the $2 \times 2$ matrix on the left must be nonnegative, so its eigenvalues must be nonnegative. After some more algebra, we can show that this implies the point $y_{G}^{\prime}+i y_{G}^{\prime \prime}$ must lie inside a definite set of circles in the complex $Y_{G}$-plane. The matrix $Z$ contains some parameters that we are free to vary, and the final bounds lie inside the convex hull generated by considering all these bounding circles. Similar statements are also possible for the quantity $y_{K}$, of course, although the results are quite a bit simpler in that case.

\subsection{Bounds on thermal expansivity $\beta^{*}$}

Now recall that

$$
\frac{1}{K^{*}+Y_{K}}=\frac{f_{A}}{K_{A}+Y_{K}}+\frac{f_{B}}{K_{B}+Y_{K}},
$$

which is closely related to "canonical function" $\Lambda(u)$, since $\Lambda\left(3 Y_{K} / 4\right) \equiv K^{*}$. Using this definition of $K^{*}$ to replace it in the earlier expression (2) for $\beta^{*}$, we find (after some more algebra) that:

$$
\beta^{*}=\langle\beta\rangle+\frac{f_{A} f_{B}\left(\beta_{B}-\beta_{A}\right)\left(K_{B}-K_{A}\right) Y_{k}}{K_{A} K_{B}+\langle K\rangle Y_{K}} .
$$

Having this formula in hand, we can take over all the results from Gibiansky and Milton (1993) directly to obtain bounds on the possible complex values of $\beta^{*}$.

Results are displayed in Figs. 2 and 3 for the example [from Gibiansky and Milton (1993)] having $\left(K_{A}, G_{A}\right)=(1+2 i, i)$ and $\left(K_{B}, G_{B}\right)=(1,1)$ (all in units of $10 \mathrm{GPa}$ ). Recall that

typical units for bulk and shear moduli are SI units of $\operatorname{GPa}\left(=10^{9} \mathrm{~N} / \mathrm{m}^{2}\right)$. Units for the constituents were chosen here to be $10 \mathrm{GPa}$ times values used in this one example from the 
Gibiansky and Milton (1993) paper, since the real parts of their chosen values are smaller than those for most solids by a factor of at least 10. Similarly, typical units for thermal expansion are: ppm $K^{-1}=10^{-6}\left({ }^{o} \mathrm{~K}\right)^{-1}$ (which is one microstrain per degree $K$ ). We have also included a factor of 10 here (so the units shown are $10^{-5}\left[{ }^{o} \mathrm{~K}\right]^{-1}$ ) again in order to bring the chosen values more in line with those for typical materials of interest. Note that material $A$ has $G_{A}=i$, so - having no real part of its shear modulus - this means we should think of it as a viscous fluid.

Figure 2 displays the steps in the bounding process: (a) Shows the Gibiansky and Milton (1993) bounds on $Y_{K}$. There are two other curves considered during the bounding process, but the ones shown are the two outer most, and therefore are the ones that are pertinent. (b) Shows the resulting bounds on the overall bulk modulus $K^{*}$ of the system for all isotropic composites composed of materials $A$ and $B$. (c) Shows the bounds on the overall thermal expansion coefficient $\beta^{*}$. Results are also shown for the coherent potential approximation (CPA) for $K^{*}$ (Soven, 1967; Gubernatis and Krumhansl, 1975; Berryman, 1980a,b; Willis, 1981), and for the center-of-mass (CM) location based on the bounding curves for $\beta^{*}$. We see that $K_{C P A}^{*}$ and $K_{C M}^{*}$ are very close to each other for the case of $f_{A}=f_{B}=0.50$ plotted here. Table 1 also displays the values for the same case but for seven choices of $f_{A}$. Figure 3 shows the bounding curves for $\beta^{*}$ for the same seven cases, plus the end points. $K_{C P A}^{*}$ is also included in each of the bounding sub-diagrams. We see that $K_{C P A}^{*}$ is not so centrally located in the other diagrams, for $f_{A} \neq 0.50$, although the issue becomes moot as $f_{A} \rightarrow 0.00$ and also as $f_{A} \rightarrow 1.00$, since the bounding regions become very small in this limit.

\section{Transversely isotropic binary composites}

Binary composites need not have isotropic symmetry overall, so results for anisotropic binary composites are also of interest.

Landau and Lifshitz (1986) show quite generally that, for anisotropic materials having triclinic, monoclinic, and orthorhombic symmetry, the thermal expansion $\beta$ has three inde-

pendent components (biaxial), while for tetragonal, rhombohedral, and hexagonal symmetry two independent components (uniaxial), and for cubic or isotropic symmetry only one component. We will now consider a transversely isotropic (or hexagonal symmetry) material such as one might obtain by layering (or laminating) two isotropic materials [see (Backus, 
1962)], with the resulting composite then being uniaxial.

\subsection{Hexagonal symmetry}

Christensen (1979) (p. 325) shows that, for a binary $(A, B)$ viscoelastic composite [note: Christensen actually discusses only elastic composites in this part of his book, but as shown here already the step from elastic to viscoelastic materials is immediate] composed of two isotropic constituents, the effective overall thermal expansion tensor coefficient $\beta^{*}$ can be written as

$$
\beta_{i j}^{*}=\delta_{i j}\langle\beta\rangle+\frac{\beta_{B}-\beta_{A}}{\frac{1}{K_{B}}-\frac{1}{K_{A}}}\left(3 S_{i j m m}^{*}-\delta_{i j}\left\langle\frac{1}{K}\right\rangle\right) .
$$

Here $S^{*}$ is the compliance tensor, repeated subscripts $m$ are summed, and the meanings

of the bracketed expressions are exactly as before. Transverse isotropy of the desired type can easily be attained in practice through a layering or lamination process, as mentioned previously.

Assuming that the axis of symmetry is $x_{3}$, then it is straightforward to show that

$$
S_{11 m m}^{*}=\delta_{m n} S_{22 m n}^{*}=\frac{1-\nu_{12}^{*}}{E_{11}^{*}}-\frac{\nu_{13}^{*}}{E_{33}^{*}}=\left(1-\frac{c_{13}^{*}}{c_{33}^{*}}\right)\left[c_{11}^{*}+c_{12}^{*}-\frac{2\left(c_{13}^{*}\right)^{2}}{c_{33}^{*}}\right]^{-1}
$$

where $E_{11}^{*}$ and $E_{33}^{*}$ are the pertinent Young's moduli, $\nu_{12}^{*}$ and $\nu_{13}^{*}$ are the pertinent Poisson's ratios, and $\beta_{11}^{*}=\beta_{22}^{*}=\beta_{\perp}^{*}$. We also find specifically that

$$
S_{33 m m}^{*}=\frac{1-2 \nu_{13}^{*}}{E_{33}^{*}}=\left(1-\frac{2 c_{13}^{*}}{c_{11}^{*}+c_{12}^{*}}\right)\left[c_{33}^{*}-\frac{2\left(c_{13}^{*}\right)^{2}}{\left(c_{11}^{*}+c_{12}^{*}\right)}\right]^{-1},
$$

which is needed for $\beta_{33}^{*}=\beta_{\|}^{*}$. For completeness, the expressions for both formulas in terms of the corresponding stiffness $c_{i j}^{*}$ matrix elements also presented in (28) and (29), as this version can be useful when measurements are obtained from acoustic or ultrasound data (Musgrave, 2003). It is easy to show that, for isotropic arrangements of the isotropic constituents, Eqs. (28) and (29) both reduce to $1 / 3 K^{*}$, since for isotropic systems we always have $(1-2 \nu) / E=$ $1 / 3 K$. Thus, (27) reduces to (2), as expected in the isotropic limit.

For the transversely isotropic case, we finally have the formulas for the two distinct $\beta^{*}$ 's:

$$
\beta_{\perp}^{*}=\langle\beta\rangle+3 \frac{\beta_{B}-\beta_{A}}{\frac{1}{K_{B}}-\frac{1}{K_{A}}}\left(\frac{1-\nu_{12}^{*}}{E_{11}^{*}}-\frac{\nu_{13}^{*}}{E_{33}^{*}}-\left\langle\frac{1}{3 K}\right\rangle\right),
$$

and

$$
\beta_{\|}^{*}=\langle\beta\rangle+\frac{\beta_{B}-\beta_{A}}{\frac{1}{K_{B}}-\frac{1}{K_{A}}}\left(\frac{3\left(1-2 \nu_{13}^{*}\right)}{E_{33}^{*}}-\left\langle\frac{1}{K}\right\rangle\right) .
$$


Note that for a medium having hexagonal symmetry, the effective overall bulk modulus is given by the Reuss average, which is:

$$
\frac{1}{K^{*}} \equiv \frac{1}{K_{R}^{*}}=\frac{2\left(1-\nu_{12}^{*}\right)}{E_{11}^{*}}+\frac{\left(1-4 \nu_{13}^{*}\right)}{E_{33}^{*}} .
$$

So we have in general that

$$
\frac{2 \beta_{\perp}^{*}}{3}+\frac{\beta_{\|}^{*}}{3}=\langle\beta\rangle+\frac{\beta_{B}-\beta_{A}}{\frac{1}{K_{B}}-\frac{1}{K_{A}}}\left(\frac{1}{K^{*}}-\left\langle\frac{1}{K}\right\rangle\right),
$$

which can be viewed as a sumrule, and as such is in agreement with (2).

Furthermore, it will prove useful to make the definitions

$$
\frac{1}{K_{\perp}^{*}} \equiv \frac{3\left(1-\nu_{12}^{*}\right)}{E_{11}^{*}}-\frac{3 \nu_{13}^{*}}{E_{33}^{*}}
$$

and

$$
\frac{1}{K_{\|}^{*}} \equiv \frac{3\left(1-2 \nu_{13}^{*}\right)}{E_{33}^{*}} .
$$

Then, (32) is also seen as the statement

$$
\frac{1}{K^{*}}=\frac{1}{3}\left(\frac{2}{K_{\perp}^{*}}+\frac{1}{K_{\|}^{*}}\right) .
$$

To derive equations (27), we first generalize (1) to

$$
\left(\begin{array}{c}
\delta \epsilon_{11} \\
\delta \epsilon_{22} \\
\delta \epsilon_{33} \\
\delta s
\end{array}\right)=\left(\begin{array}{cccc}
S_{1111} & S_{1122} & S_{1133} & \beta_{\perp} \\
S_{2211} & S_{2222} & S_{2233} & \beta_{\perp} \\
S_{3311} & S_{3322} & S_{3333} & \beta_{\|} \\
\beta_{\perp} & \beta_{\perp} & \beta_{\|} & c_{p}^{*} / T
\end{array}\right)\left(\begin{array}{c}
\delta \sigma_{11} \\
\delta \sigma_{22} \\
\delta \sigma_{33} \\
\delta \theta
\end{array}\right),
$$

with the understanding that the two constituents themselves have the same equations as before, but these can be telescoped out into this same form as shown in (37) by noting that the $\beta$ 's are all the same for each constituent, and that $S_{1111}^{A}=1 / E_{A}, S_{1122}^{A}=-\nu_{A} / E_{A}$, etc.

We consider two thought experiments this time. One for the strains in the horizontal planes: $\delta \sigma_{\perp}=\delta \sigma_{11}=\delta \sigma_{22}$, and a second one for the strains along the axis of symmetry $\delta \sigma_{\|}=\delta \sigma_{33}$. In each case, the stresses must be uniform, but not necessarily the same in both cases, so $\delta \sigma_{\perp}=\delta \sigma_{11}=\ldots=\delta \sigma_{33}$ and $\delta \sigma_{\|}=\delta \sigma_{11}=\ldots=\delta \sigma_{33}$. But in general for anisotropic media, the stresses pertinent to the argument are such that $\delta \sigma_{\perp} \neq \delta \sigma_{\|}$. Then we have

$$
\delta \epsilon_{\perp}=S_{11 m m}^{*} \delta \sigma_{\perp}+\beta_{\perp}^{*} \delta \theta=\left(\frac{1-2 \nu_{A}}{E_{A}}\right) \delta \sigma_{\perp}+\beta_{A} \delta \theta=\left(\frac{1-2 \nu_{B}}{E_{B}}\right) \delta \sigma_{\perp}+\beta_{B} \delta \theta .
$$


Also, since $1 / 3 K_{A}=\left(1-2 \nu_{A}\right) / E_{A}$, the equations in (38) are completely analogous to the ones shown previously for the isotropic case - except that they differ by a factor of 3 , since $\delta \epsilon_{\perp}$ is not the total strain, but only one of the three linear components of total strain. And similarly for the thermal expansion along the axis of symmetry, we also have

$$
\delta \epsilon_{\|}=S_{33 m m}^{*} \delta \sigma_{\|}+\beta_{\|}^{*} \delta \theta=\left(\frac{1-2 \nu_{A}}{E_{A}}\right) \delta \sigma_{\|}+\beta_{A} \delta \theta=\left(\frac{1-2 \nu_{B}}{E_{B}}\right) \delta \sigma_{\|}+\beta_{B} \delta \theta .
$$

In fact, the only difference (except for labeling) between (38) and (39) is the replacement of $S_{11 m m}^{*}$ by $S_{33 m m}^{*}$. We can conclude by making the analogy between the anisotropic and the isotropic cases more precise using the the definitions (34) and (35), if desired.

Again, microstructure does not enter formulas (30) and (31) explicitly, but the influence of the microstructure is felt directly through the overall Young's moduli $E_{11}^{*}$ and $E_{33}^{*}$ as well as through the corresponding Poisson's ratios in both equations.

\subsection{Averaging thin layers at low frequencies}

Backus (1962) presents a very elegant method of determining the effective constants for a layered medium composed of thin isotropic or anisotropic elastic layers. As we shall see, his method also works equally well for viscoelastic layers. For simplicity and to be consistent with the earlier parts of the paper, we assume here that the layers are all isotropic. If $K_{A}$ and $G_{A}$ are the bulk and shear moduli of component $A$, then the Lamé constant $\lambda_{A} \equiv K_{A}-2 G_{A} / 3$. Similar definitions then apply to constituent B. The Voigt $6 \times 6$ notation for the stiffness matrix then takes the form:

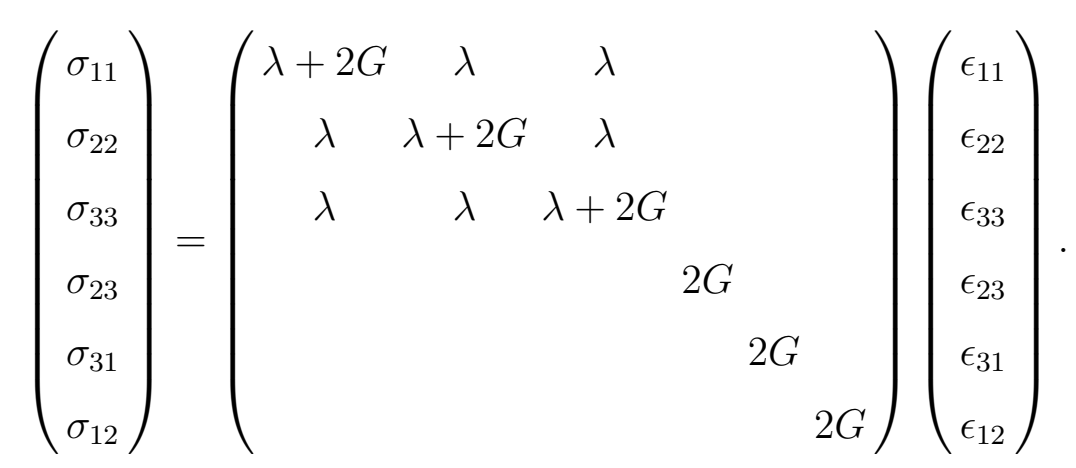

We have chosen to define the strain tensor here in terms of the particle displacements $u_{i}$ according to

$$
\epsilon_{i j}=\frac{1}{2}\left(u_{i, j}+u_{j, i}\right) \equiv \frac{1}{2}\left(\frac{\partial u_{i}}{\partial x_{j}}+\frac{\partial u_{j}}{\partial x_{i}}\right)
$$


[This is not the only choice possible, and in fact it is not the most common one used in the seismology community; these two choices differ by a factor of two for the cases when $i \neq j$. We make this choice here to simplify the eigenvalue structure of the resulting stiffness matrix.] The key step in Backus's (1962) analysis [also see Milton (2002)] is to find a rearrangement of (40) such that the rapidly varying coefficients multiply slowly varying stresses or trains. Following this procedure, we find:

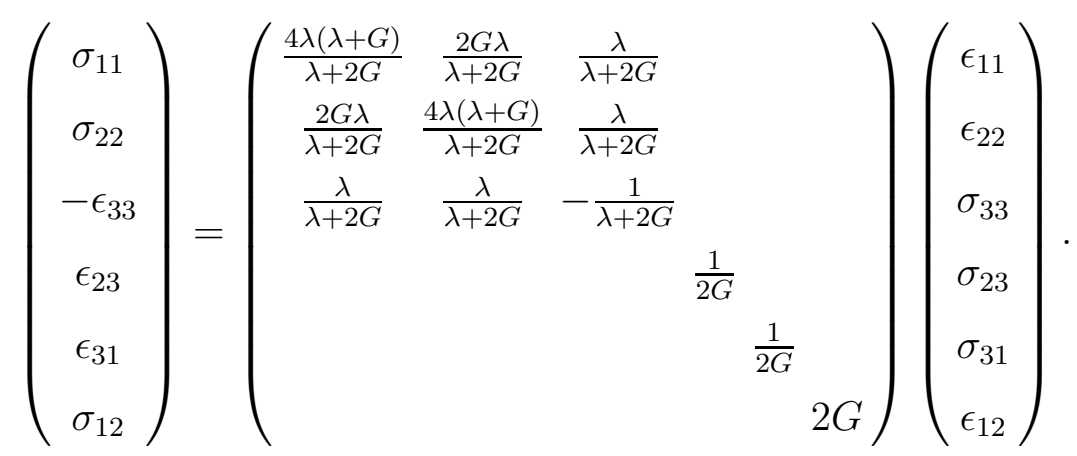

The vector on the left contains the fast variables. The vector on the right contains the slow variables, which (at sufficiently low frequencies) may be treated as constants. So (42) can now be averaged essentially by inspection (Berryman, 1999). The result is:

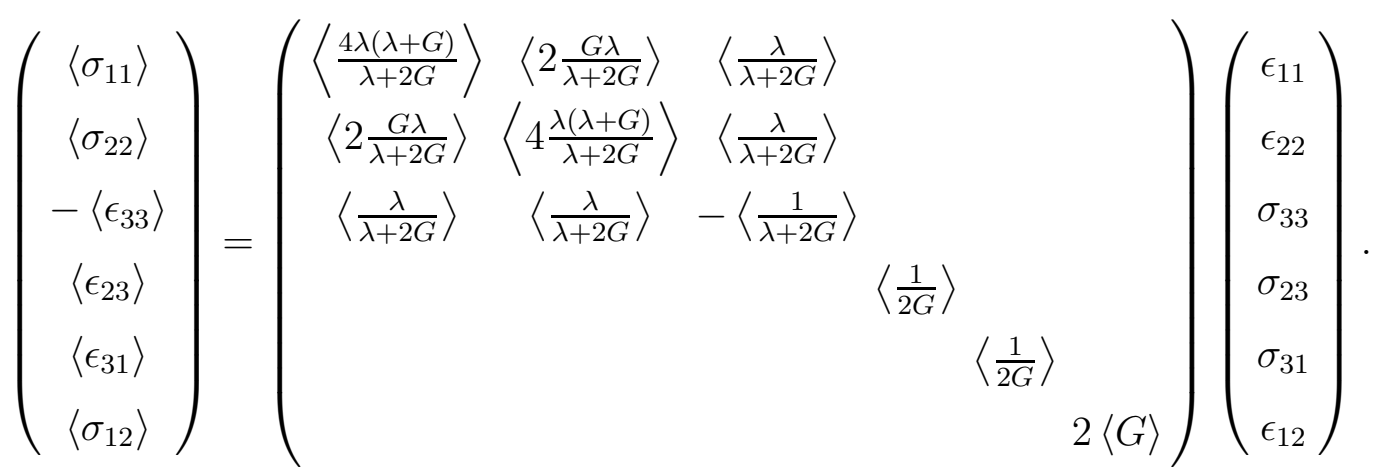

The relationships among these averages and the stiffnesses of the hexagonal system are given explicitly by:

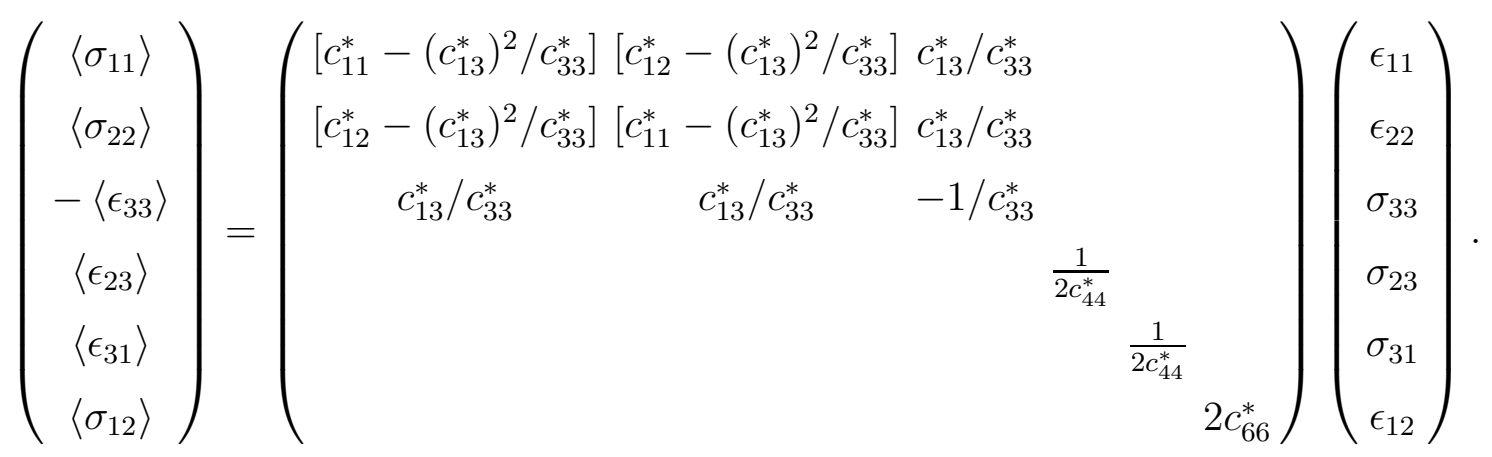

Equations (43) and (44) can be unraveled to yield formulas for the stiffnesses $c_{i j}^{*}$ associated with the principal stresses in the anisotropic medium. The results are then given by the 
formulas:

$$
\begin{gathered}
c_{33}^{*}=\left\langle\frac{1}{\lambda+2 G}\right\rangle^{-1}, \\
c_{11}^{*}=c_{33}^{*}\left\langle\frac{\lambda}{\lambda+2 G}\right\rangle^{2}+4\left\langle\frac{G(\lambda+G)}{\lambda+2 G}\right\rangle, \\
c_{12}^{*}=c_{33}^{*}\left\langle\frac{\lambda}{\lambda+2 G}\right\rangle^{2}+2\left\langle\frac{G \lambda}{\lambda+2 G}\right\rangle,
\end{gathered}
$$

and

$$
c_{13}^{*}=c_{33}^{*}\left\langle\frac{\lambda}{\lambda+2 G}\right\rangle .
$$

The remaining two formulas for $c_{44}$ and $c_{66}$ are not required for the application under consideration here, but are included nevertheless for the sake of completeness. These formulas are:

$$
c_{44}^{*}=\left\langle\frac{1}{G}\right\rangle^{-1}
$$

and

$$
c_{66}^{*}=\langle G\rangle,
$$

which are respectively the harmonic mean and the mean based on volume fraction averages) of the shear moduli for the two constituents $A$ and $B$.

The most important point about the analysis just shown (and the reason for repeating it here) is to emphasize that nowhere are there any energy arguments used, only arguments based on simple algebra. Thus, the formulas apply equally well to elastic or viscoelastic media. All the coefficients and formulas relating them to constituent properties may therefore be correctly treated as complex.

\subsection{Eigenvalues of the complex stiffness matrix}

It may not be obvious to some readers that the complex stiffness matrix will have complex eigenvalues, and that these eigenvalues must have non-negative imaginary parts in order for the analysis to make physical sense (otherwise negative dissipation would necessarily ensue,

which implies the absurd situation in which the system is generating energy without any need for external sources). The way to check for the presence or absence of this conceptual problem is to evaluate the imaginary parts of all the complex eigenvalues of the complex 
stiffness matrix and determine their signs. Four of these are very simple, and occur in pairs:

$$
\gamma_{3}=2 c_{44}=2\left\langle\frac{1}{G}\right\rangle^{-1}
$$

and

$$
\gamma_{4}=2 c_{66}=2\langle G\rangle
$$

Eigenvalue $\gamma_{3}=2 c_{44}$ appears explicitly twice in the equation for the case of hexagonal symmetry:

$$
\left(\begin{array}{l}
\sigma_{11} \\
\sigma_{22} \\
\sigma_{33} \\
\sigma_{23} \\
\sigma_{31} \\
\sigma_{12}
\end{array}\right)=\left(\begin{array}{llllll}
c_{11} & c_{12} & c_{13} & & & \\
c_{12} & c_{11} & c_{13} & & & \\
c_{13} & c_{13} & c_{33} & & & \\
& & & 2 c_{44} & & \\
& & & & 2 c_{44} & \\
& & & & & 2 c_{66}
\end{array}\right)\left(\begin{array}{l}
\epsilon_{11} \\
\epsilon_{22} \\
\epsilon_{33} \\
\epsilon_{23} \\
\epsilon_{31} \\
\epsilon_{12}
\end{array}\right),
$$

while $\gamma_{4}$ appears once explicitly, and once implicitly because of the transverse isotropy condition $c_{11}-c_{12}=2 c_{66}=\gamma_{4}$. The remaining two eigenvalues are determined by a quadratic characteristic equation, having the solutions:

$$
\gamma_{ \pm}=\frac{1}{2}\left[c_{11}+c_{12}+c_{33} \pm \sqrt{\left(c_{11}+c_{12}-c_{33}\right)^{2}+8 c_{13}^{2}}\right] .
$$

The $c_{i j}$ 's for the present problem are given explicitly by the formulas quoted already in (45)-(50). For the plus sign in (54), we have $\gamma_{1} \equiv \gamma_{+} \simeq 3 K^{*}$, by which we mean that this eigenvalue acts somewhat like 3 times the bulk modulus at intermediate values of volume fraction, and reduces exactly to 3 times the unique bulk modulus as $f_{A} \rightarrow 0.00$ or 1.00. Similarly, $\gamma_{2} \equiv \gamma_{-} \simeq 2 G^{*}$, by which we mean this eigenvalue acts somewhat like 2 times the shear modulus at intermediate values of $f_{A}$, and reduces exactly to twice the unique shear modulus at both volume fraction end points.

Figure 4 displays the results of these computations. It is clear that all six eigenvalues of complex stiffness exhibit non-negative imaginary parts, and therefore non-negative dissipation (as is expected on physical grounds - i.e., energy conservation, or energy loss coupled with an increase of entropy). 


\subsection{Examples of $\beta_{\perp}^{*}$ and $\beta_{\|}^{*}$}

Figure 5 illustrates the results for $\beta_{\perp}^{*}$ and $\beta_{\|}^{*}$ based on Eqs. (30) and (31), using the right hand sides of (28) and (29), together with (45)-(50) for the effective stiffness coefficients. Note that the imaginary parts of $K_{R}^{*}$ and $K_{\perp}^{*}$ are all positive, whereas the sign of the imaginary parts of the $\beta^{*}$ s are mixed, as are the imaginary parts of $K_{\|}^{*}$. The dotted line at zero has been added as an aid to the eye, to show where the imaginary part of $1 / K_{\|}^{*}$ becomes positive. Recall that Figure 4 shows all the eigenvalues of the complex stiffness matrix have non-negative dissipation, so these observed changes of sign in the complex thermal expansion coefficients are not related to any unphysical behavior of the model. Figure 4 also demonstrates however that this behavior, although unusual, is not unphysical as the eigenvalues themselves always have non-negative imaginary parts - so it is impossible to excite separately any unphysical combination of these specially defined moduli. The lesson seems to be that it is best to write all the moduli in terms of the eigenvalues and the modes of excitation in terms of the eigenvectors, so as not to permit unnecessary confusion in interpretation to arise in this context. This leads us to Section 4 where we study the eigenvalues of the complex coupled system in more detail. We find that the mixed behavior of the imaginary parts of the $\beta$ 's does not affect the anticipated result that the dissipation will be non-negative for all the modes of the coupled system.

\section{Eigenvalues of the coupled system}

Now we have already shown that viscoelastic part of the overall system of equations has non-negative dissipation at all times. It is however still not obvious whether the fully coupled system, including both the thermal expansion and heat capacity effects, also has this property. In fact, we have not checked this issue for the isotropic problem yet either. We will do this first for the $2 \times 2$ system (1), to show explicitly the types of steps needed and then we will repeat the process for the more complicated $4 \times 4$ system.

\subsection{Eigenvalues for the $2 \times 2$ system}

The first difficulty with studying the eigenvalues of these coupled systems is the fact that the components of the $2 \times 2$ matrix typically have inconsistent units. So it is necessary to 
transform this problem to one having either no units (i.e., dimensionless), or to one having uniform (consistent) units. There is more than one way to achieve the desired result, and we will present two of the choices here.

First, Biot (1956) has considered the thermoelastic problem, and treated both the anisotropic and isotropic cases. He chose to express the change in entropy as

$$
\delta s \equiv \frac{\delta h}{T}=\beta K \delta \epsilon+c_{v} \delta \theta / T
$$

where $\delta h$ is the increment of heat, $c_{v}$ is heat capacity at constant volume, and the reference temperature is $T$ for the unstrained/unstressed sample.

Then, the thermoelastic relations for the (hexagonal) anisotropic problem can be written as

$$
\left(\begin{array}{c}
\sigma_{11} \\
\sigma_{22} \\
\sigma_{33} \\
-\delta s
\end{array}\right)=\left(\begin{array}{cccc}
c_{11} & c_{12} & c_{13} & -\beta_{\perp} K \\
c_{12} & c_{11} & c_{13} & -\beta_{\perp} K \\
c_{13} & c_{13} & c_{33} & -\beta_{\|} K \\
-\beta_{\perp} K & -\beta_{\perp} K & -\beta_{\|} K & -c_{v} / T
\end{array}\right)\left(\begin{array}{c}
\epsilon_{11} \\
\epsilon_{22} \\
\epsilon_{33} \\
\delta \theta
\end{array}\right) .
$$

For the isotropic problem, this equation reduces to

$$
\left(\begin{array}{c}
-\delta p \\
-\delta s
\end{array}\right)=\left(\begin{array}{cc}
K & -3 \beta K \\
-3 \beta K & -c_{v} / T
\end{array}\right)\left(\begin{array}{l}
\delta \epsilon \\
\delta \theta
\end{array}\right),
$$

where - as before - we have $-\delta p \equiv\left(\sigma_{11}+\sigma_{22}+\sigma_{33}\right) / 3$, and $\delta \epsilon \equiv \epsilon_{11}+\epsilon_{22}+\epsilon_{33}$. We note that $\delta p$ and $\delta h$ [the increment of heat from (55)] have the same dimensions. Strain $\epsilon$ is dimensionless, as is the ratio $\delta \theta / T$. So multiplying the bottom row of the matrix (and also the left-hand vector) by $T$, and then multiplying the right-hand column of the matrix by $T$, and dividing $\delta \theta$ by $T$ gives the following result:

$$
\left(\begin{array}{c}
-\delta p \\
-T \delta s
\end{array}\right)=\left(\begin{array}{cc}
K & -3 \beta K T \\
-3 \beta K T & -c_{v} T
\end{array}\right)\left(\begin{array}{c}
\delta \epsilon \\
\delta \theta / T
\end{array}\right) .
$$

Similar transformations can be performed on (56) with similar looking results. We find in both cases that the transformed equation has a right-hand vector that is dimensionless, a left-hand vector that has dimensions of pressure, and a matrix that also has consistent dimensions of a pressure. The eigenvalues $q_{ \pm}$from the quadratic characteristic equation are:

$$
q_{ \pm}=\frac{1}{2}\left[K-c_{v} T \pm \sqrt{\left(K+c_{v} T\right)^{2}-36(\beta K T)^{2}}\right]
$$


It is of course possible to rewrite (2) in a similar fashion, but it will be important to arrange the matrix for the isotropic case so the upper left hand corner remains unchanged as $1 / K$. To accomplish this, we arrive at the following transformed equation:

$$
\left(\begin{array}{c}
\delta \epsilon / K \\
T \delta s / K^{2}
\end{array}\right)=\left(\begin{array}{cc}
1 / K & 3 \beta T / K \\
3 \beta T / K & c_{p} T / K^{2}
\end{array}\right)\left(\begin{array}{c}
-\delta p / K \\
\delta \theta / T
\end{array}\right) .
$$

The vector on the right is now dimensionless. The vector on the left has dimensions of inverse pressure. The transformed matrix now has dimensions of compliance (which is also inverse of pressure). So we have arrived at another consistent form, although it is not quite as simple as the one for Biot's formulation. Also, it is impotant to recognize that these two matrices are not simply inverses of each other, as the choices of pairings of the independent and dependent variables are different.

It is straightforward now to find the two complex eigenvalues $\left(Q_{ \pm}\right)$of this transformed $2 \times 2$ matrix. The results that follow from the characteristic quadratic equation are:

$$
Q_{ \pm}=\frac{1}{2 K^{2}}\left(K+c_{p} T \pm \sqrt{\left(K-c_{p} T\right)^{2}+36(\beta K T)^{2}}\right) .
$$

This form is natural in the sense that, if the off-diagonal elements (proportional to $\beta$ ) vanish, the results are just the diagonal elements of the matrix as expected.

We will next show some examples of these results for the isotropic case in Figure 6. We need numerical values for $c_{p}$ and for $T$. A typical value for $T$ is nominal room temperature, which is $T \simeq 300^{\circ} \mathrm{K}$. For water, the value of $c_{p} \simeq 4 \times 10^{6} \mathrm{~N} / \mathrm{m}^{2 o} \mathrm{~K}$, or $c_{p} \simeq 4 \mathrm{MPa} /{ }^{o} \mathrm{~K}$. A typical value for rubber is $c_{p} \simeq 2 \mathrm{MPa} /{ }^{\circ} \mathrm{K}$, which is the value we will use in the examples. So $c_{p} T \simeq 600 \mathrm{MPa}=0.6 \mathrm{GPa}$, at room temperature.

\subsection{Eigenvalues for the $4 \times 4$ system}

Next, we seek a formula that is automatically of the right type to permit complex eigenvalue analysis and that is also a straightforward generalization of (60). We find that the $4 \times 4$ transformed matrix that replaces $(60)$ for hexagonal symmetry is:

$$
\left(\begin{array}{c}
\delta \epsilon_{11} / K \\
\delta \epsilon_{22} / K \\
\delta \epsilon_{33} / K \\
\tau \delta s / K
\end{array}\right)=\left(\begin{array}{cccc}
S_{1111} & S_{1122} & S_{1133} & \beta_{\perp} \tau \\
S_{1122} & S_{1111} & S_{1133} & \beta_{\perp} \tau \\
S_{1133} & S_{1133} & S_{3333} & \beta_{\|} \tau \\
\beta_{\perp} \tau & \beta_{\perp} \tau & \beta_{\|} \tau & \chi \tau
\end{array}\right)\left(\begin{array}{c}
\delta \sigma_{11} / K \\
\delta \sigma_{22} / K \\
\delta \sigma_{33} / K \\
\delta \theta / \tau K
\end{array}\right),
$$


where (in order to simplify the notation) we introduced the definitions $\tau=T / K^{\prime}$, and $\chi=c_{p} / K^{\prime}$, where $K^{\prime}=\operatorname{Re}\left(K_{R}\right)$. [This choice differs from the one made in (60). And other choices are also possible as well. Some further discussion of this point will be given later together with the examples. But the main reason for these choices is that the order of the resulting eigenvalue problem in (60) was only 2, and was therefore easily solved using the quadratic formula. However, Eqn. (62) is fourth order. As we shall soon see, it is easily reduced to a cubic equation. But, the complication of having complex normalization factors such as $K_{R}$ makes it more difficult than seems necessary to the application (e.g., keeping track of these three complex branches as $f_{B}$ changes seems unnecessarily tedious). However, this problem goes away altogether when we introduce a real factor such as $K^{\prime}=\operatorname{Re}(K)$ in place of the complex $K_{R}$ itself.] We also made use of the symmetries: $S_{1111}=S_{2222}$, $S_{1122}=S_{2211}$, and $S_{1133}=S_{2233}=\ldots$, etc. This equation will now be analyzed to determine the natures of the four resulting eigenvalues.

First, it is easy to see that one of the four eigenvector/eigenvalue pairs is trivial, since $\delta \sigma_{11}=-\delta \sigma_{22}$ is an obvious shear mode, and also an eigenvector of the combined system with eigenvalue $S_{1111}-S_{1122}=1 / 2 c_{66} \equiv Q_{4}$. This fact will allow us to reduce the size of the eigenvalue problem from the $4 \times 4$ shown to a $3 \times 3$ system. This result is most easily accomplished by considering the determinant corresponding to the characteristic equation for the eigenvalues $Q$, which is

$$
\left|\begin{array}{cccc}
S_{1111}-Q & S_{1122} & S_{1133} & \beta_{\perp} \tau \\
S_{1122} & S_{1111}-Q & S_{1133} & \beta_{\perp} \tau \\
S_{1133} & S_{1133} & S_{3333}-Q & \beta_{\|} \tau \\
\beta_{\perp} \tau & \beta_{\perp} \tau & \beta_{\|} \tau & \chi \tau-Q
\end{array}\right|=0 .
$$

We can proceed with some standard manipulations of the determinental form.

First, subtract the second column from the first column; then, the second row from the first row. Follow this by multiplying the resulting first row by $\frac{1}{2}$ and adding it to the second row. Then, multiplying the resulting first column by $\frac{1}{2}$, and adding it to the second column. 
The resulting equation for the determinant is now:

$$
\left|\begin{array}{cccc}
2\left(S_{1111}-S_{1122}-Q\right) & 0 & 0 & 0 \\
0 & \frac{1}{2}\left(S_{1111}+S_{1122}-Q\right) & S_{1133} & \beta_{\perp} \tau \\
0 & S_{1133} & S_{3333}-Q & \beta_{\|} \tau \\
0 & \beta_{\perp} \tau & \beta_{\|} \tau & \chi \tau-Q
\end{array}\right|=0 .
$$

Thus, both the first column and row can now be eliminated while noting that $\left(S_{1111}-S_{1122}-\right.$ $Q=0$ is exactly the equation of the shear mode having $Q=Q_{4}$ that we already discussed. The $3 \times 3$ system determinant is now:

$$
\left|\begin{array}{ccc}
\left(S_{1111}+S_{1122}-Q\right) & 2 S_{1133} & 2 \beta_{\perp} \tau \\
S_{1133} & S_{3333}-Q & \beta_{\|} \tau \\
\beta_{\perp} \tau & \beta_{\|} \tau & \chi \tau-Q
\end{array}\right|=0
$$

where we have multiplied the top row of the determinant (65) by a factor of 2 in order to put this expression into the form of an eigenvalue problem for the reduced $3 \times 3$ matrix. This form provides the cubic equation that determines the three nontrivial eigenvalues $Q_{1}$, $Q_{2}$, and $Q_{3}$ that we seek.

Even in the absence of the frequency dependent thermal expansion effects, the remaining modes of this system are not usually simple. Common modes such as $\delta \sigma_{11}=\delta \sigma_{22}=\delta \sigma_{33}$ which is a simple uniform pressure (and might therefore result in a uniform contraction or expansion for an isotropic system) - and $\delta \sigma_{11}=\delta \sigma_{22}=-\frac{1}{2} \delta \sigma_{33}$ - which is a type of shear stress (the author calls this the "uniaxial shear stress") are not eigenmodes of an anisotropic elastic (or viscoelastic) system. Without coupling through thermal expansion, we would have quasi-compressional and quasi-shear modes each of which have mixed physical character, and often not easily interpreted. This situation is further complicated by the presence of the heat capacity and thermal expansion effects included here. Indeed, if eigenvalue $Q_{1}$ corresponds to something like a quasi-compressional mode, and $Q_{2}$ corresponds to a quasi-shear mode, then presumably we can also speak of the eigenvalue $Q_{3}$ corresponding to a quasi-thermal mode - being mostly a change of the heat content due to a temperature change, but also having some coupling to the stress-strain changes through the presence of the thermal expansion coefficients.

Our point is that there is not likely to be any further simplification of the eigenvalue/eigenvector structure for this problem. The equation of interest that needs to be 
solved is (63). We could write this out as a third-order polynomial in $Q$, but all the information is contained in (63), and perhaps it is in its most interpretable form already. So we will not provide any further details of the analysis (see some important details of the solution process presented in Appendix A), and go straight to the examples.

Results for the three nontrivial eigenvalues for the anisotropic case are presented in Figure 7. Figs. 7(a) and 7(b) show two different views of the same results for the eigenvalues $Q_{1}$, $Q_{2}$, and $Q_{3}$ from (65). The results show clearly that the two eigenvalues associated with inverse compressional $\left.Q_{1}\right)$ and shear $\left(Q_{2}\right)$ moduli - but including coupling through the complex thermal expansion coefficients, still have negative imaginary parts, which translate to positive dissipation in all cases. The third eigenvalue is actually associated with the complex extension of the heat capacity $c_{p}$; the positive imaginary part of this eigenvalue is also associated with positive dissipation. Recall that the mixed character of the matrix we study here means that two of the eigenvalues are related to compliances, while the third related to the heat capacity, which is more properly thought of as a stiffness (since those are its natural units). So opposite signs of the imaginary parts are expected. [A physical stiffness should have positive imaginary part, while a physical compliance has negative imaginary part. $Q_{3}$ has dimensions of a compliance due to our normalizations, but the pertinent physical quantity $c_{p}$ itself has dimensions of stiffness. Some of these conceptual complications could have been avoided by using Biot's formulation (56). We will leave this easier case as an exercise for the reader.]

Fig. 7(c) shows the real and imaginary parts of the inverses of the first two complex eigenvalues $Q_{1}^{-1}$ and $Q_{2}^{-1}$ [again from (65)] plotted versus the volume fraction $f_{B}$. Fig. $7(\mathrm{~d})$ shows the same two eigenvalues plotted in the complex plane. These two figures may be compared directly with Figs. 4(a) and 4(c), since the units are the same and the examples shown here also act approximately like $3 \times$ the quasi-bulk modulus and $2 \times$ the quasi-shear modulus, respectively.

\section{Applications}

The methods presented have a variety of applications from fluid-fluid mixtures to fluidsolid suspensions, and from fluid-saturated porous media to viscoelastic solid-solid composites. 
If the viscoelastic media being mixed are fluids (so $\operatorname{Re} G=0$ for both constituents), and if furthermore both constituents have $\operatorname{Im} G=0$, then we have an exact result for the bulk modulus of the isotropic system, which is

$$
K^{*}=\langle K\rangle^{-1}=\left(\frac{f_{A}}{K_{A}}+\frac{f_{B}}{K_{B}}\right)^{-1} .
$$

Furthermore, the same result also holds for the layered case, as it is well-known that Backus's (1962) formulas reduce to Hill's (1963) equation if the shear modulus is the same in both constituents; and, since both are zero in the situation under discussion, Hill's equation reduces to the harmonic mean. Substituting (66) into (2) then produces the appropriate formula for $\beta^{*}$.

If the viscoelastic medium is a fluid-solid suspension, that implies that the fluid is the dominant component and the solids (think of them as particles) are suspended in the fluid. The fluid component will always have $\operatorname{Re} G=0$ (by definition), but fluids can in principle be viscous (water, oil) or nonviscous (low temperature liquid helium is one example). So $\operatorname{Im} G_{\text {fluid }}=0$ may or may not hold, depending on the nature of the suspending fluid. For the nonviscous fluid, $G_{\text {fluid }} \equiv 0$, and this case reduces essentially to the previous one having (66) as the exact result for the overall bulk modulus of the system. Substituting (66) into (2) again produces the appropriate formula for $\beta^{*}$. But, when the fluid is viscous, the overall behavior is more complicated and some effective medium theory [such as the one discussed by Berryman (1980b)] might be a better approach for estimating the effective $K^{*}$ to use in (2). Note that all the examples in this paper have $G_{A}=i$, making constituent $A$ a viscous fluid.

A fluid suspension model of the type just described might be a useful model as well of the glass transition in glass-forming liquids (Dyre, 2006). In fact, it has been noted recently that the specific heat $c_{p}$ should itself be $\omega$ dependent in glass-forming liquids (Christensen et al., 2007), so it is perhaps not surprising to be finding, as we have done here, that the thermal expansion coefficient can also be frequency dependent.

Fluid-saturated porous media have microgeometries opposite to the preceding case, having fluid on the inside and solid acting as container. This situation always has $\operatorname{Re} G^{*} \neq 0$, since this is the defining characteristic of a solid. The situation is complicated further by the fact that the external boundary conditions make a difference to the results. If the porous medium is fully drained, the fluid can move in and out freely, and has little effect on the 
overall behavior. Then, the key bulk modulus in the system is the overall drained bulk modulus $K_{d}^{*}$ (which can be complex). If however the fluid is trapped or experiences undrained boundary conditions, then the pertinent overall bulk modulus will be $K_{u}^{*} \equiv$ the undrained bulk modulus, which for a nonviscous fluid (or for very low frequency behavior) is determined by Gassmann's (1951) equation. See Coussy (2004) for a discussion of the thermoporoelastic case. Then, the present work leads naturally to a discussion of the extension to thermoporoviscoelastic cases. For viscous fluids or higher frequencies, the behavior of such systems requires further study to determine the correct value of $K_{u}^{*}$. There is ongoing work in this general area of study concerning bulk moduli for both isotropic and anisotropic porous media, as is exemplified by the recently published work of Giraud et al. (2007).

If the viscoelastic composite is a solid-solid composite, then we have the case that the majority of readers were probably considering throughout most of this paper. In this situation, the shear moduli of both constituents are nonzero and both might be viscoelastic. The overall bulk modulus $K^{*}$ of the system is then strongly dependent on the composite microstructure, and the bounding methods described in section 2 are the most general way to analyze these problems. The alternative is to estimate $K^{*}$ for particular microstructures. For design purposes, this is the recommended approach. For analysis of data such as ultrasound or seismic data, effective medium theories (Berryman, 1980a;b) can play a useful role again.

In all these cases it is important to consider how the thermal expansion affects the overall response, and especially so at higher frequencies.

\section{Discussion and conclusions}

Another important point to discuss is the fact that the relationships (2), (27), (30), and (31) between the thermal expansivities $\beta^{*}, \beta_{\perp}^{*}, \beta_{\|}^{*}$ and the bulk moduli $K^{*}$ of the overall composite system are general, and apply to any type of microstructure as long as the true system symmetry (isotropic, hexagonal) is appropriate for the formulas quoted. If in addition we know the microstructure, as in the case of layered or laminated media, then it is also possible to obtain formulas for the overall bulk moduli $K^{*}$ and, therefore, formulas for the thermal expansivities $\beta_{\perp}^{*}$ and $\beta_{\|}^{*}$ as well. However, in the case of isotropic media, there exists

literally an infinite variety of available microstructures. So, in these cases, one very useful 
approach (rather than trying to enumerate a very small subset of the many possibilities) is to provide general bounds over all possible microstructures. This was the approach pursued here.

Another approach that was briefly mentioned in the examples is to make use of effective medium theories, such as the coherent potential approximation (CPA) (Soven, 1967; Gubernatis and Krumhansl, 1975; Berryman, 1982), or the differential scheme (DS) (Norris, 1985; Garboczi and Berryman, 2001), to provide specific estimated values for complex overall $K^{*}$. Because these two specific theories are known to have definite microstructures associated with them (Milton, 1985; Avellaneda, 1987), they are (at least in principle) realizable and therefore the values will always lie inside the rigorous bounds. Those microstructures may however not always be realistic representations of the microstructure of interest in a particular application. Nevertheless, in some cases these values may be of direct interest, especially if the actual microstructure of the systems of interest does somehow mimic that of the effective medium models — which has been shown to happen sometimes in practice (Berge $e t$ al., 1993; Berryman and Berge, 1996).

To illustrate the differences in the overall bulk modulus $K^{*}$ for the isotropic and hexagonal (here due to layering) symmetries, Figure 8 plots the results for the effective $K^{*}$ for the layered problem together with five of the bounding regions for the isotropic examples. We see that these bounding regions do overlap the unique curve for the layered example. So the bulk moduli in both situations are somewhat comparable, and not disjoint. In contrast, the harmonic mean $K_{h}$ of the constituent moduli is also plotted and we see that this estimator differs significantly from both $K_{R}^{*}$ and the bounding regions for the isotropic composite media. A reasonable conclusion is that $K_{h}$ is not an appropriate estimator for these problems, whereas $K_{R}^{*}, K_{C P A}^{*}$, and a few other effective medium estimates such as those for the differential scheme, may be appropriate estimators for these viscoelastic constants.

Once good estimates of the overall bulk modulus $K^{*}$ are known, then the formulas for the overall thermal expansion coefficient $\beta^{*}$ provide an excellent tool for determining the time and frequency dependent behavior of composite viscoelastic media.

A final point concerns the fact that, although we have shown the eigenvalues of those coupled systems considered here have proper behavior, i.e., the dissipation is always nonnegative, it would nevertheless be desirable (but necessarily beyond our current scope) to prove that this result is always true for such systems. This line of inquiry will for now be 
left to future work.

\section{Acknowledgments}

This work is dedicated to Graeme W. Milton on the occasion of receiving the William Prager Medal from the Society of Engineering Science for his work in solid mechanics. Work performed under the auspices of the U.S. Department of Energy by the University of California, Lawrence Berkeley National Laboratory under Contract No. DE-AC03-76SF00098 and supported specifically by the Geosciences Research Program of the DOE Office of Basic Energy Sciences, Division of Chemical Sciences, Geosciences and Biosciences.

\section{Appendix A: Solving the complex cubic eigenvalue problem}

The eigenvalue problem resulting from the determinental equation (65) is the following cubic equation:

$$
F(Q) \equiv\left(Q-Q_{1}\right)\left(Q-Q_{2}\right)\left(Q-Q_{3}\right)=Q^{3}-Q^{2} S+Q C-P=0
$$

where the coefficients are given explicitly by

$$
\begin{gathered}
S=Q_{1}+Q_{2}+Q_{3}=S_{1111}+S_{1122}+S_{3333}+\chi \tau \\
C=Q_{2} Q_{3}+Q_{3} Q_{1}+Q_{1} Q_{2}=\left[\left(S_{1111}+S_{1122}\right) S_{3333}-2 S_{1133}^{2}\right] \\
+\chi \tau\left(S_{1111}+S_{1122}+S_{3333}\right)-\left(2 \beta_{\perp}^{2}+\beta_{\|}^{2}\right) \tau^{2}
\end{gathered}
$$

and

$$
\begin{aligned}
P=Q_{1} Q_{2} Q_{3} & =\chi \tau\left[\left(S_{1111}+S_{1122}\right) S_{3333}-2 S_{1133}^{2}\right] \\
& -\beta_{\|}^{2} \tau^{2}\left(S_{1111}+S_{1122}\right)+4 \beta_{\|} \beta_{\perp} \tau^{2} S_{1133}-2 \beta_{\perp}^{2} \tau^{2} S_{3333} .
\end{aligned}
$$

We refer to these three coefficients respectively as the sum $(S)$, the cross check $(C)$, and the product $(P)$ for the cubic equation.

One technical point concerning the solution of the resulting complex cubic equation should be elaborated. It is straightforward to set up this problem as a Newton-Raphson iteration scheme (Hildebrand, 1974; Press et al., 1988):

$$
q_{i+1}=q_{i}-F\left(q_{i}\right) / F^{\prime}\left(q_{i}\right)
$$


where $q_{i}$ is the most recent iterate $i$ (or a starting value for $i=0$ ), and $F^{\prime}$ is the first derivative of the cubic polynomial. But it turns out that only one of the three distinct roots is found easily this way. The solution of the entire cubic can then be found using one step of deflation, i.e., reducing the size of the problem one level from cubic to quadratic. We can easily solve the remaining quadratic equation for the two remaining roots once we have found the most stable root of the cubic using the Newton-Raphson iterative procedure. If $q_{\text {final }}$ is the result of the finitely terminated Newton-Raphson process, then the deflated equation for the quadratic problem remaining to be solved is:

$$
Q^{2}-Q\left(S-q_{\text {final }}\right)+\frac{P}{q_{\text {final }}}=0 .
$$

We can check that the first result $q_{\text {final }}$ is actually one of the three roots by performing a cross check showing that

$$
q_{\text {final }}\left(S-q_{\text {final }}\right)+\frac{P}{q_{\text {final }}} \rightarrow C,
$$

to the desired level of precision.

Equation (72) is a second order equation for finding the two remaining roots, and it can be solved using the quadratic formula. The only remaining difficulty is that the continuity of the results for plotting purposes can be problematic in the kinds of displays shown here when the volume fractions $\left(f_{B}\right)$ are changing. It is important to distinguish (and properly identify) the three roots. To do so we must make a decision at each step as to which of the solutions of the quadratic should be associated with which one of the three roots. The author found this part of the problem became relatively straightforward when the factors used to normalize (i.e., create uniform units in) the matrix were all real. Then, it was sufficient to assign the eigenvalues so the ones that had the same signs (one will be positive, the other negative) for the imaginary part of the square root of the solution of the quadratic equation. However, there did not appear to be a simple solution to this problem if the normalizing factors were chosen to be complex. For then the complex character of $K$ interacted with the other complex factors present in a way not easy to disentangle; so this is why real factors are recommended in the text. The author tried three choices of these factors: $1 / \operatorname{Re}(K)$, $\operatorname{Re}(1 / K)$, and $1 / \operatorname{Abs}(K)=\operatorname{Abs}(1 / K)$. All three choices gave very similar - though not identical - results in the form of curves that were equally simple to plot and interpret. So, there was not a very strong reason to choose one of these over the other two possibilities listed. It was however observed that the choice $1 / \operatorname{Re}(K)$ gave the smoothest curves, and 
this characteristic seemed to indicate better analytical behavior than that of the other two choices mentioned. 


\section{References}

Angell, C. A., Ngai, K. L., McKenna, G. B., McMilan, P. F., Martin, S. W., 2000. Relaxation in in glass-forming liquids and amorphous solids. J. Appl. Phys. 88, 3113-3157.

Avellaneda, M., 1987. Iterated homogenization, differential effective medium theory and applications. Commun. Pure Appl. Math. 40, 527-554.

Backus, G. E., 1962. Long-wave elastic anisotropy produced by horizontal layering. J. Geophys. Res. 67, 4427-4440.

Benveniste, Y., Dvorak, G. J., 1990. On a correspondence between mechanical and thermal effects in two-phase composites. In Micromechanics and Inhomogeneity: The Toshio Mura 65th Anniversary Volume, edited by G. J. Weng, M. Taya, and H. Abé, SpringerVerlag, New York, pp. 65-81.

Benveniste, Y., Dvorak, G. J., 1992. Uniform-fields and universal relations in piezoelectric composites. J. Mech. Phys. Solids, 40 (6), 1295-1312.

Berge, P. A., Berryman, J. G., Bonner, B. P., 1993. Influence of microstructure on rock elastic properties. Geophys. Res. Lett. 20, 2619-2622.

Berryman, J. G., 1980a. Long-wavelength propagation in composite elastic media I. Spherical inclusions. J. Acoust. Soc. Am. 68, 1809-1819.

Berryman, J. G., 1980b. Long-wavelength propagation in composite elastic media II. Ellipsoidal inclusions. J. Acoust. Soc. Am. 68, 1820-1831.

Berryman, J. G., 1982. Effective medium theory for elastic composites. In Elastic Wave Scattering and Propagation, edited by V. K. Varadan and V. V. Varadan, Ann Arbor Science, Ann Arbor, Michigan, pp. 111-129.

Berryman, J. G., 1999. Transversely isotropic elasticity and poroelasticity arising from thin isotropic layers. In Theoretical and Computational Acoustics 199\% edited by Y.C. Tengx, E.-C. Shang, Y.-H. Pao, M. H. Schultz, and A. D. Pierce, World Scientific, Singapore, pp. 457-474. 
Berryman, J. G., Berge, P. A., 1996. Critique of explicit schemes for estimating elastic properties of multiphase composites. Mech. Materials 22, 149-164.

Berryman, J. G., Milton, G. W., 1991, Exact results for generalized Gassmann's equations in composite porous media with two constituents, Geophysics 56, 1950-1960.

Berryman, J. G., Milton, G. W., 1992, Exact results in linear thermomechanics of fluidsaturated porous media, Appl. Phys. Lett. 61, 2030-2032.

Biot, M. A., 1956. Thermoelasticity and irreversible thermodynamics. J. Appl. Phys. 27, $240-253$.

Christensen, R. M., 1979, Mechanics of Composite Materials, Wiley Interscience, New York, pp. 31-72.

Christensen, R. M., 1982, Theory of Viscoelasticity, Dover Publications, Mineola, New York, pp. $138-140$.

Christensen, T., Olsen, N. B., Dyre, J. C., 2007. Conventional methods fail to measure $c_{p}(\omega)$ of glass-forming liquids. Phys. Rev. E 75, 041502.

Coussy, O., 2004. Poromechanics. John Wiley and Sons, Hoboken, NJ., pp. 84-87.

Cribb, J. L., 1968. Shrinkage and thermal expansion of a two phase material, Nature 220, $576-577$.

Dvorak, G. J., 1990. On uniform-fields in heterogeneous media, Proc. Royal Soc. London A, 431, 89-110.

Dvorak, G. J., Benveniste, Y., 1992. On transformation strains and uniform-fields in multiphase elastic media. Proc. Royal Soc. London A 437, 291.

Dvorak, G. J., Benveniste, Y., 1997. On micromechanics of inelastic and piezoelectric composites. In Theoretical and Applied Mechanics 1996, edited by T. Tatsumi, E. Watanabe, and T. Kambe, Elsevier Science, Amsterdam, pp. 65-81.

Dyre, J. C., 2006. Colloquium: The glass transition and elastic models of glass-forming liquids. Rev. Mod. Phys. 78, 953-972. 
Fei, Y., 1995. Thermal expansion. In Mineral Physics and Crystallography: A Handbook of Physical Constants, edited by T. J. Ahrens, American Geophysical Union, Washington, D.C., vol. 2, pp. 29-44.

Ferry, J. D., 1970. Viscoelastic Properties of Polymers. John Wiley and Sons, New York, pp. $140-141$.

Garboczi, E. J., Berryman, J. G., 2001. New differential effective medium theory for the linear elastic moduli of a material containing composite inclusions. Mech. Materials 33, 455-470.

Gassmann, F., 1951. Über die elastizität poröser medien. Veirteljahrsschrift der Naturforschenden Gesellschaft in Zürich 96, 1-23.

Gibiansky, L. V., Milton, G. W., 1993. On the effective viscoelastic moduli of two-phase media. I. Rigorous bounds on the complex bulk modulus. Proc. Roy. Soc. London A 440, 163-188.

Giraud, A., Huynh, Q. V., Hoxha, D., Kondo, D., 2007. Effective poroelastic properties of transversely-isotropic rock-like composites with arbitrarily oriented ellipsoidal inclusions. Mech. Mater. 39 (11), 1006-1024.

Grabovsky, Y., Milton, G. W., Sage, D. S., 2000. Exact relations for effective tensors of composites: Necessary conditions and sufficients conditions. Commun. Pure Appl. Math. 53 (3), 300-353.

Gubernatis, J. E., Krumhansl, J. A., 1975. Macroscopic engineering properties of polycrystalline materials: Elastic properties. J. Appl. Phys. 46, 1875-1883.

Hashin, Z., Shtrikman, S., 1963. A variational approach to the theory of the elastic behavior of multiphase materials. J. Mech. Phys. Solids 11, 127-140.

Hestholm, S., Ketcham, S., Greenfield, R., Moran, M., McMechan, G., 2006. Quick and accurate (Q) parameterization in viscoelastic wave modeling. Geophysics 71, T147T150.

Hildebrand, F. E., 1974. Introduction to Numerical Analysis. Dover Publications, New York, pp. 575-630. 
Hill, R., 1963. Elastic properties of reinforced solids: Some theoretical principles. J. Mech. Phys. Solids 11, 357-372.

Hsieh, C.-L., Tuan, W.-H., 2006. Elastic and thermal expansion behavior of two-phase composites. Mat. Sci. Engineering A 425, 349-360.

Hsieh, C.-L., Tuan, W.-H., 2007. Thermal expansion behavior of a model ceramic-metal composite. Mat. Sci. Engineering A 460-461, 453-458.

Lakes, R. S., 1999. Viscoelastic Solids. CRC Press, Boca Raton, Florida, pp. 294-299.

Landau, L. D., Lifshitz, E. M., 1986. Theory of Elasticity. Butterworth Heineman, Oxford, p. 36.

Levin, V. M., 1967. Thermal expansion coefficients of heterogeneous materials. Mech. Solids $2,58-61$.

Milton, G. W., 1985. The coherent potential approximation is a realizable effective medium scheme. Comm. Math. Phys. 99, 463-500.

Milton, G. W., 1987. Multicomponent composites, electrical networks, and new types of continued fraction. I. Commun. Math. Phys. 111, 281-327.

Milton, G. W., 2002. The Theory of Composites. Cambridge University Press, Cambridge, UK, pp. 159-163.

Milton, G. W., and Berryman, J. G., 1997. On the effective viscoelastic moduli of two-phase media. II. Rigorous bounds on the complex shear modulus in three dimensions. Proc. Roy. Soc. London A 453, 1849-1880.

Musgrave, M. J. P., 2003. Crystal Acoustics: Introduction to the Study of Elastic Waves and Vibrations in Crystals. Acoustical Society of America, Leesdale, Pennsylvania, pp. $175-185$.

Norris, A. N., 1985. A differential scheme for the effective moduli of composites. Mech. Mater. 4, 1-16. 
Press, W. H., Flannery, B. P, Teukolsky, S. A., Vetterling, W. T., 1988. Numerical Recipes in C: The Art of Scientific Computing, Cambridge University Press, Cambridge, UK, pp. $270-277$.

Reuss, A., 1929. Berechung der Fliessgrenze von Mischkristallenx auf Grund der Plastizitätsbedingung für Einkristalle. Z. Angew. Math. Mech. 9, 49-58.

Rosen, B. W., Hashin, Z., 1970. Effective thermal expansion coefficients and specific heats of composite materials. Int. J. Engng. Sci. 8, 157-173.

Schmalholz, K., Podladchikov, Y., 1999. Buckling versus folding: Importance of viscoelasticity. Geophys. Res. Lett. 26, 2641-2644.

Schulgasser, K., 1989. Thermal expansion of polycrystals. J. Material Sci. Lett. 8, 228-229.

Soven, P., 1967. Coherent-potential model of substitutional alloys. Phys. Rev. 156, 809-813.

Vinogradov, V., Milton, G. W., 2005. The total creep of viscoelastic composites under hydrostatic or antiplane loading. J. Mech. Phys. Solids 53, 1248-1279.

Voigt, W., 1928. Lehrbuch der Kristallphysik, Teubner, Leipzig.

Walpole, L. J., 1969. On the overall elastic moduli of composite materials. J. Mech. Phys. Solids 17, 235-251.

Willis, J. R., 1981. Variational and related methods for the overall properties of composites. In Advances in Applied Mechanics, edited by C.-S. Yih, Academic Press, New York, pp. $1-78$. 
TABLE 1. Effective medium theory (coherent potential approximation) values for the bulk $K^{*}$ and shear $G^{*}$ moduli, as well as for the complex thermal expansion coefficient $\beta^{*}$, of the two component composite considered in the text for some selected values of the (dimensionless) volume fraction $f_{A}$. For comparison, the center-of-mass points $\beta_{C M}^{*}$ of each bounding region obtained using the Gibiansky and Milton (1993) theory are also listed.

\begin{tabular}{|c|c|c|c|c|}
\hline \hline$f_{A}$ & $K_{C P A}^{*}(10 \mathrm{GPa})$ & $G_{C P A}^{*}(10 \mathrm{GPa})$ & $\beta_{C P A}^{*}\left(10^{-5}\left[{ }^{o} K\right]^{-1}\right)$ & $\beta_{C M}^{*}\left(10^{-5}\left[{ }^{o} K\right]^{-1}\right)$ \\
\hline 0.01 & $1.01410+i 1.96140$ & $0.01894+i 0.99890$ & $1.98451-i 0.00649$ & $1.98839-i 0.00718$ \\
0.08 & $1.09773+i 1.71914$ & $0.14321+i 0.98460$ & $1.88366-i 0.04671$ & $1.90578-i 0.05217$ \\
0.22 & $1.20339+i 1.32970$ & $0.35910+i 0.92587$ & $1.70880-i 0.10341$ & $1.73741-i 0.11483$ \\
0.50 & $1.25675+i 0.74134$ & $0.69974+i 0.71052$ & $1.41619-i 0.14336$ & $1.41767-i 0.14392$ \\
0.78 & $1.16619+i 0.28738$ & $0.93043+i 0.37323$ & $1.16744-i 0.10051$ & $1.15595-i 0.08200$ \\
0.92 & $1.07156+i 0.09713$ & $0.99137+i 0.14992$ & $1.05724-i 0.04513$ & $1.05178-i 0.03175$ \\
0.99 & $1.00976+i 0.01161$ & $1.00034+i 0.01982$ & $1.00685-i 0.00630$ & $1.00618-i 0.00405$ \\
\hline \hline
\end{tabular}




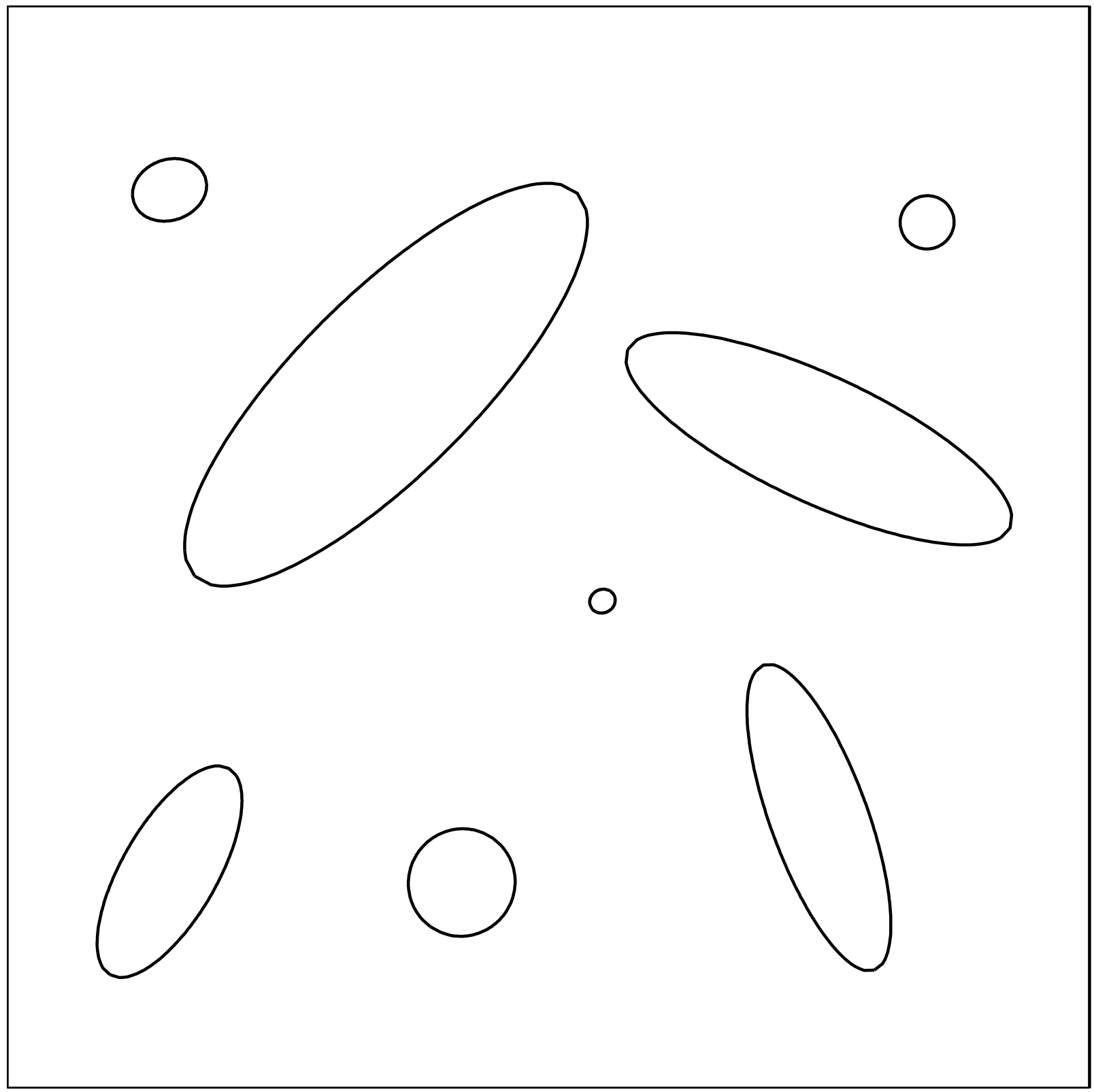

FIG. 1: Illustrating a cross-section through a statistically isotropic binary composite in which ellipsoids of one viscoelastic material are imbedded in another viscoelastic material. The theory presented does not depend however on the shape of the inclusions. 
(a)

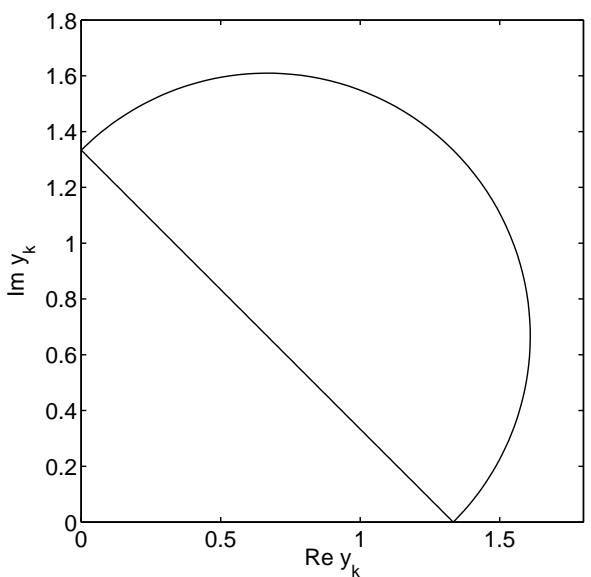

(b)

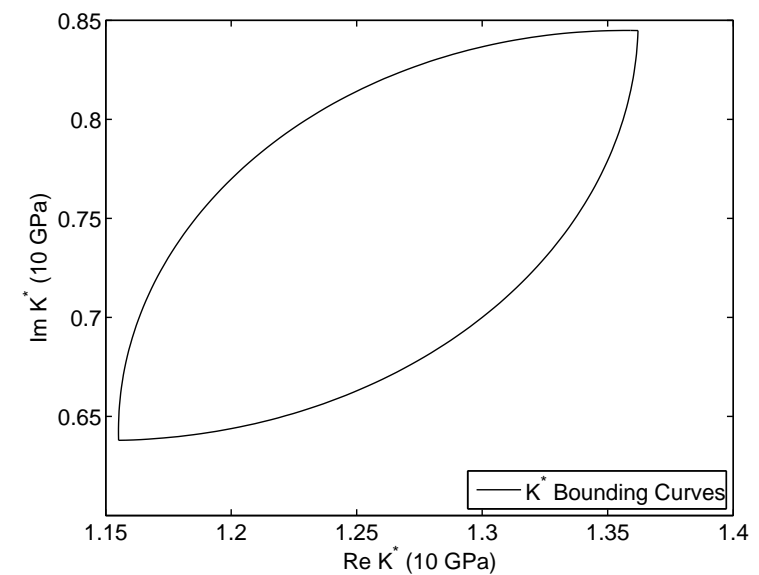

(c)

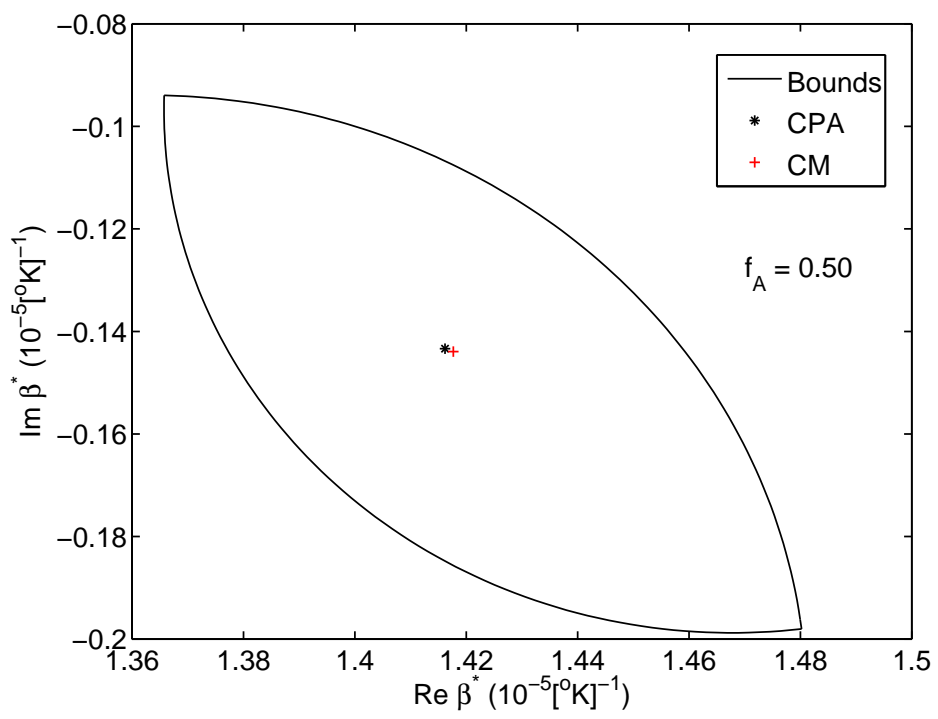

FIG. 2: Figure (a) shows the bounding curves that are given by the Gibiansky and Milton (1993) analysis for complex bulk modulus $K^{*}$, when $\left(K_{A}, G_{A}\right)=(1+2 i, i)$ and $\left(K_{B}, G_{B}\right)=(1,1)$ (all in units of $10 \mathrm{GPa}$ ). These bounds are phrased in terms of the complex $y_{K}$-transform as discussed in the text, and are independent of the volume fraction. Also see Figure 3 of Gibiansky and Milton (1993). Figure (b) shows the corresponding bounds on the actual complex bulk modulus $K^{*}$ itself for the case $f_{A}=0.50$. Figure (c) shows the resulting bounds for the complex thermal expansion $\beta^{*}$ when $\beta_{A}=1.0, \beta_{B}=2.0$ (both in units of $10^{-5}\left[{ }^{o} K\right]^{-1}$ ), and the volume fractions are $f_{A}=f_{B}=\frac{1}{2}$. Also plotted are the values of $\beta_{C P A}^{*}$ for the coherent potential approximation estimate of $\beta^{*}$, as well as the estimate obtained by computing the center-of-mass of the bounding curves themselves $\beta_{C M}^{*}$ 


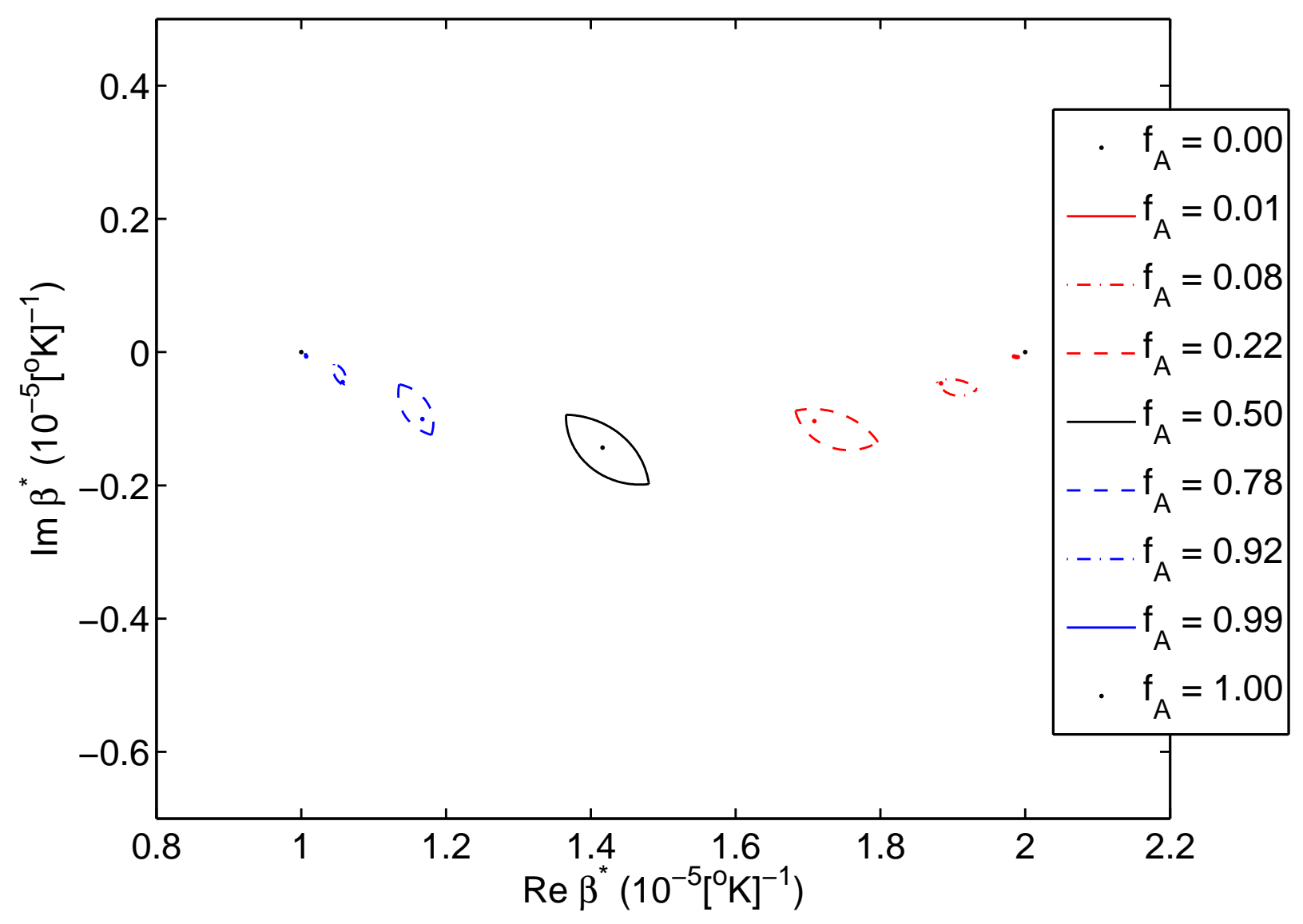

FIG. 3: Illustrating bounds (the closed curves) and CPA estimates (the dots) for the complex thermal expansion coefficient $\beta^{*}$ as the value of the volume fraction $f_{A}$ changes. When $f_{A}=0.0$, $\beta^{*}=\beta_{B}=2.0$; when $f_{A}=1.0, \beta^{*}=\beta_{A}=1.0$. The bounding region is largest when $f_{A}=0.5$, and smallest as $f_{A} \rightarrow 0.0$, or $f_{A} \rightarrow 1.0$. We will call this the "necklace diagram" as the individual bounding regions resemble gem stones, and the array of these regions resembles a display of gems on a necklace. There are obviously an infinite number of the bounding regions (as $f_{A}$ changes continuously) that could be plotted, but we have chosen a very small and select subset to display for the sake of clarity. 
(a)

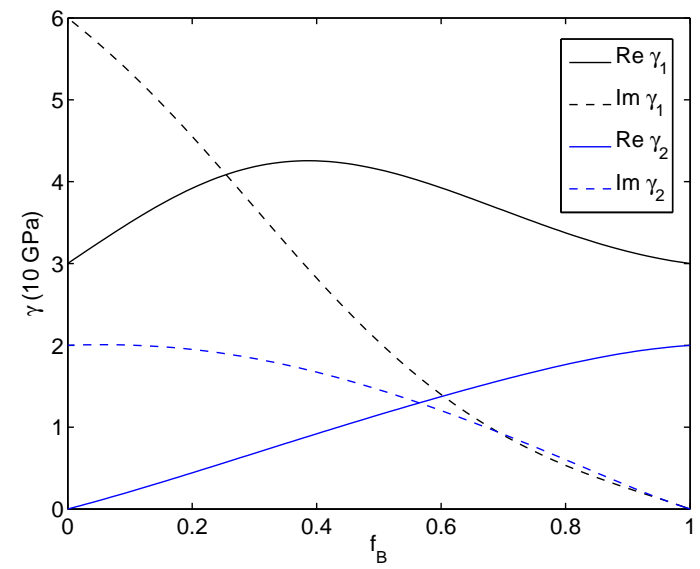

(b)

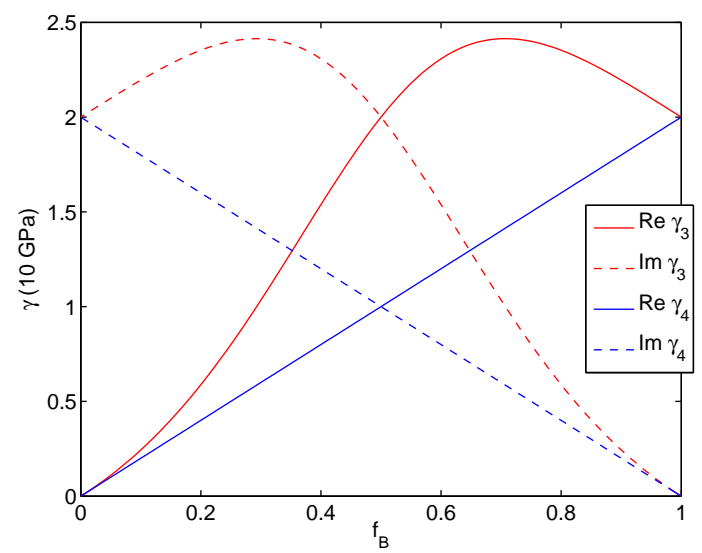

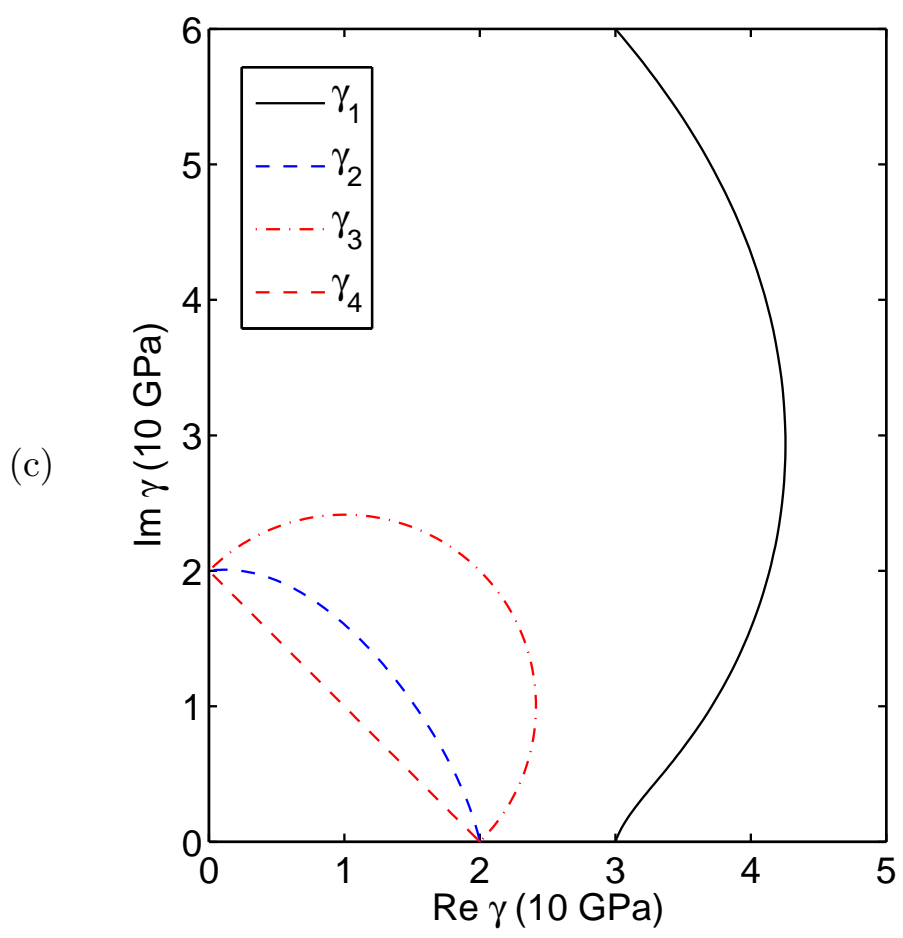

FIG. 4: Figure (a) shows the first two complex eigenvalues $\gamma_{1}=\gamma_{+}$and $\gamma_{2}=\gamma_{-}$from (53) and (54) plotted versus the volume fraction $f_{B}$. Figure (b) shows the remaining two distinct complex eigenvalues $\gamma_{3}$ and $\gamma_{4}$ plotted versus the volume fraction $f_{B}$. These two eigenvalues both have multiplicity 2 in the layered medium model. Figure (c) shows the same information contained in (a) and (b), but plotted instead in the complex plane. The main purpose of this entire figure is to illustrate that all the stiffness eigenvalues of the examples in the text have non-negative imaginary parts, and therefore non-negative dissipation. 

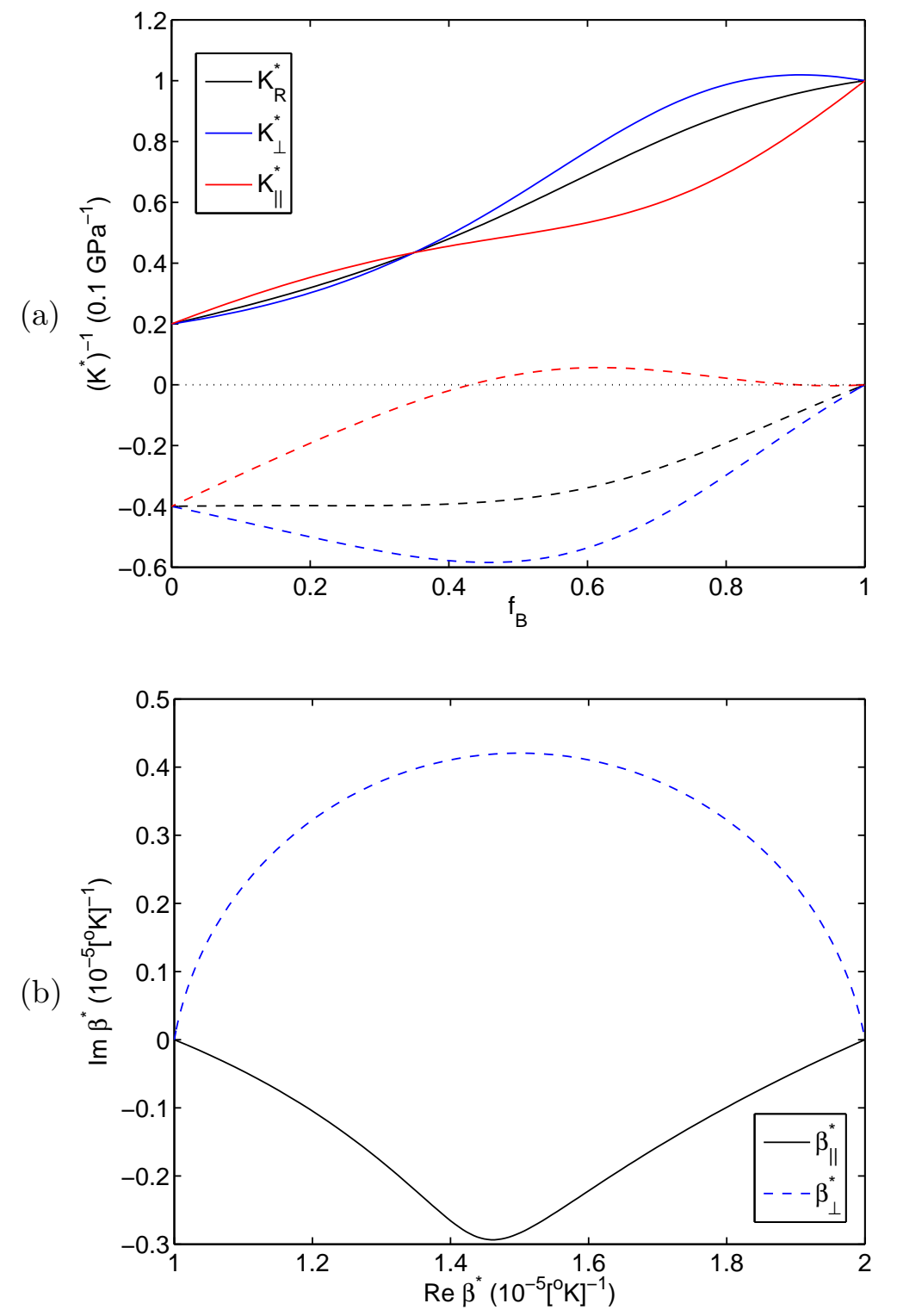

FIG. 5: Figure (a) plots the real (solid lines) and imaginary (dashed lines) parts of the inverse complex bulk modulus $K_{R}^{*}$ for a layered system as a function of $f_{B}$, as well as the same for $K_{\perp}^{*}$ and $K_{\|}^{*}$ as defined in the text. The layer properties $(\mathrm{A}, \mathrm{B})$ are the same as in previous figures. Figure (b) shows the estimates obtained for the two complex thermal expansion coefficients $\beta_{\|}^{*}$ and $\beta_{\perp}^{*}$. Note that the imaginary parts of $K_{R}^{*}, K_{\perp}^{*}$, and are all positive, whereas the imaginary parts of the $\beta^{*}$ 's are mixed, as are the imaginary parts of $K_{\|}^{*}$. The dotted line at zero has been added as an aid to the eye, to show where the imaginary part of $1 / K_{\|}^{*}$ becomes positive. Figure 4 shows that all the eigenvalues of the complex stiffness matrix have non-negative dissipation, so these observed changes of sign in the complex thermal expansion coefficients are not related to any unphysical behavior of the model. 
(a)

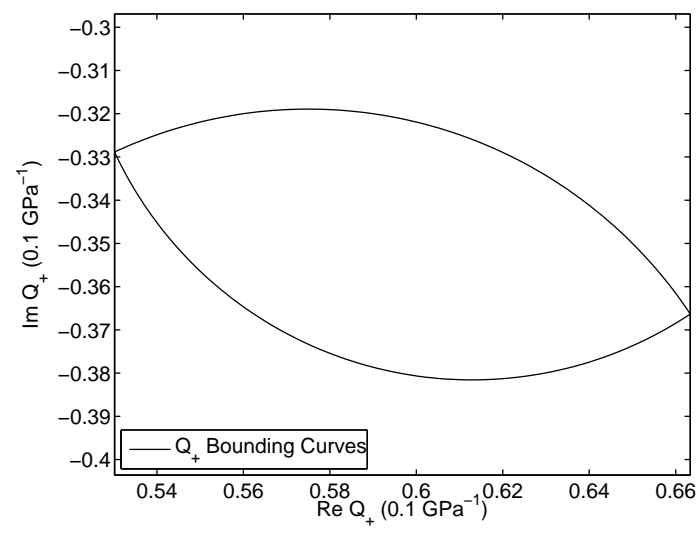

(b)

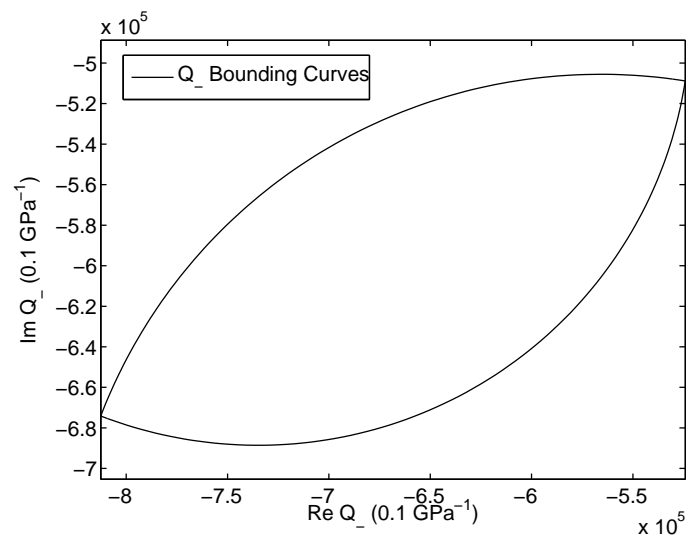

(c)

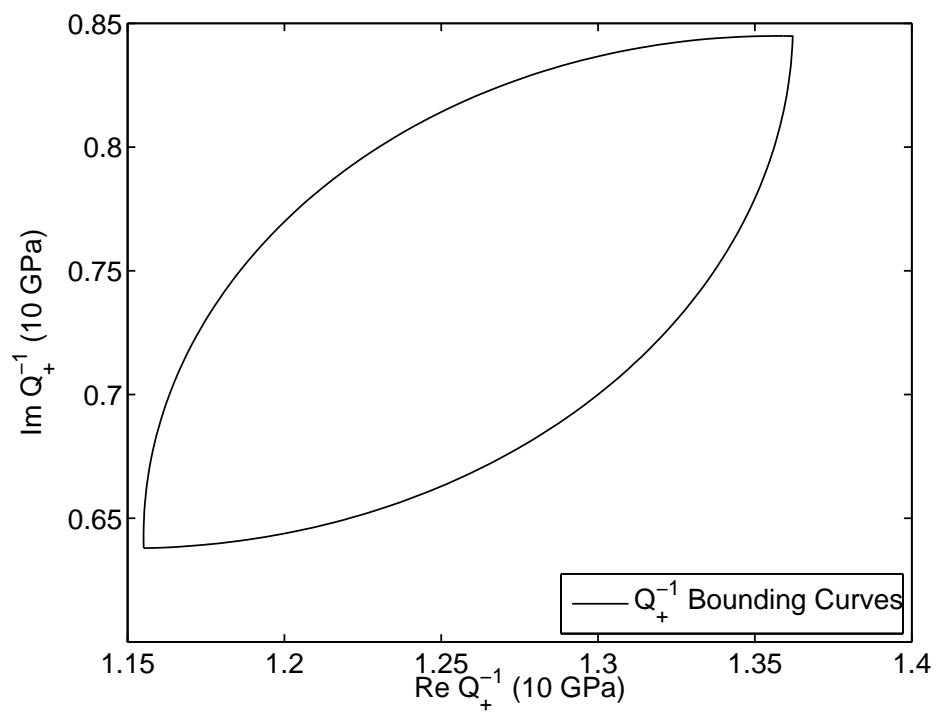

FIG. 6: Figure (a) shows the bounding curves that are given by the Gibiansky and Milton (1993) analysis for the complex eigenvalue $Q_{+}$from Eq. (61), when $\left(K_{A}, G_{A}\right)=(1+2 i, i)$ and $\left(K_{B}, G_{B}\right)=(1,1)$ (all in units of $\left.10 \mathrm{GPa}\right)$. The volume fractions here are $f_{A}=f_{B}=0.5$. These bounds correspond to the curves for bulk modulus $K^{*}$ in Figure 2. Figure (b) shows the bounds on eigenvalue $Q_{-}$, also from (61). Figure (c) shows the bounds on $1 / Q_{+}$, which may be usefully compared to the bounds in Fig. 2(b), since for weak coupling the two quantities $\left(K^{*}\right.$ and $\left.1 / Q_{+}\right)$ become nearly identical. 
(a)

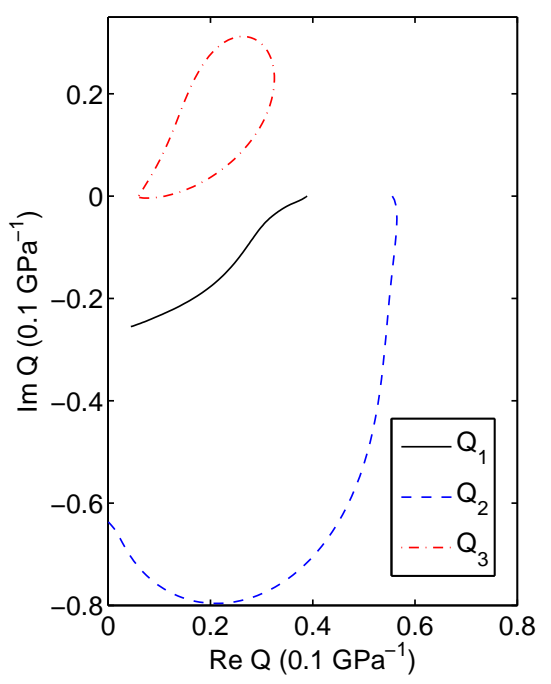

(c)

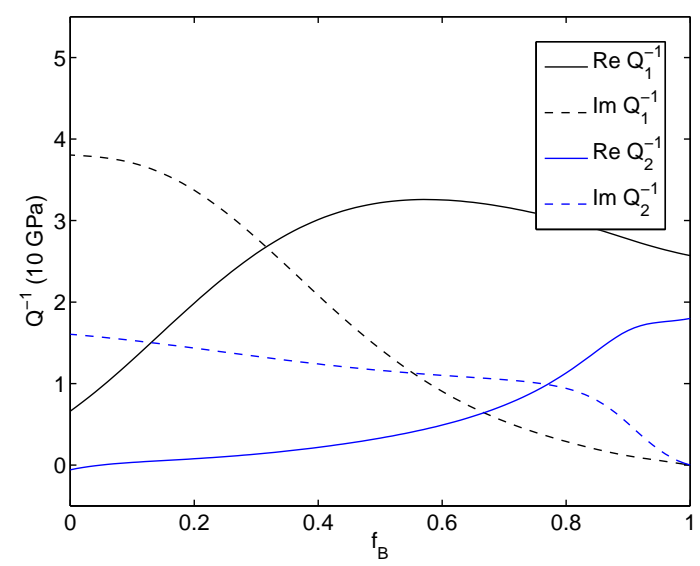

(b)

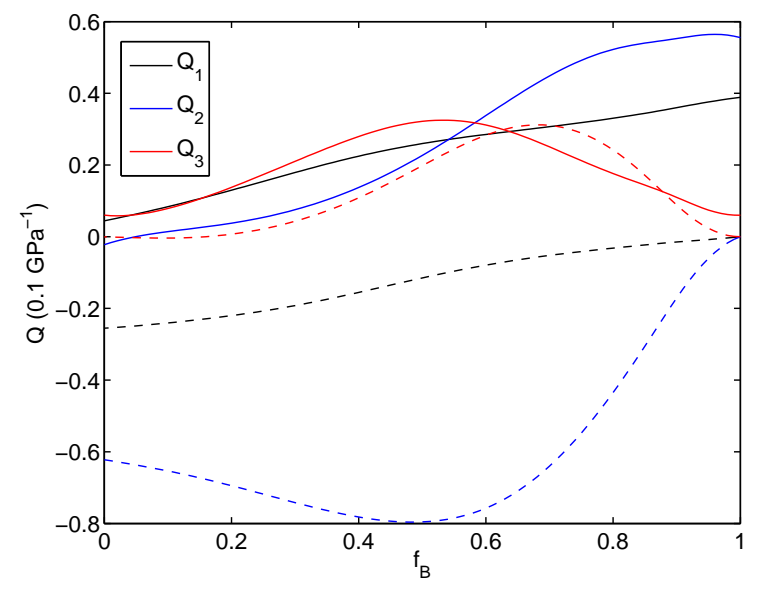

(d)

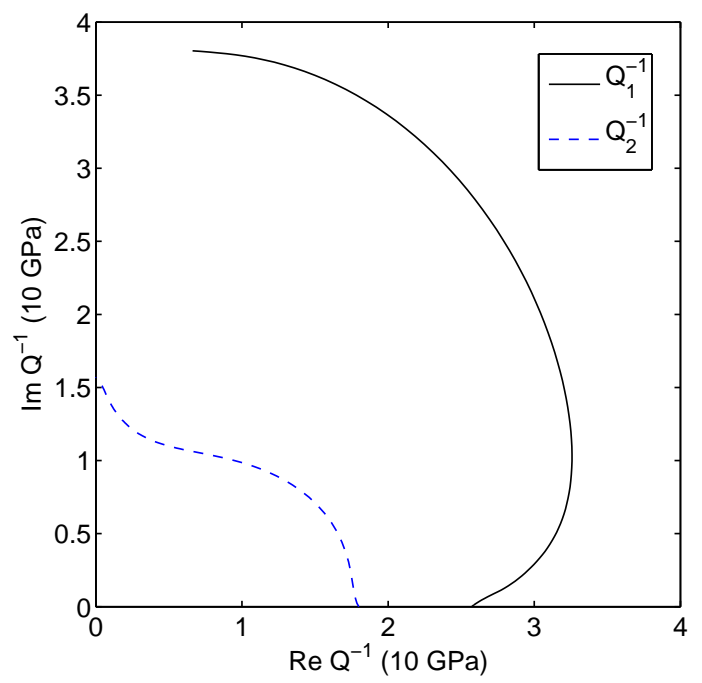

FIG. 7: Figures (a) and (b) show two different views of the same results for the eigenvalues $Q_{1}$, $Q_{2}$, and $Q_{3}$ from (65). The results show clearly that the two eigenvalues associated with inverse compressional $\left.Q_{1}\right)$ and shear $\left(Q_{2}\right)$ moduli - but including coupling through the complex thermal expansion coefficients, still have negative imaginary parts, which translate to positive dissipation in all cases. The third eigenvalue $\left(Q_{3}\right)$ is actually associated with the complex extension of the heat capacity $c_{p}$; the positive imaginary part of this eigenvalue is also associated with positive dissipation. Figure (c) shows the real and imaginary parts of the inverses of the first two complex eigenvalues $Q_{1}^{-1}$ and $Q_{2}^{-1}$ [again from (65)] plotted versus the volume fraction $f_{B}$. Figure (d) shows the same two eigenvalues plotted in the complex plane. These two figures can be compared directly with Figs. 4(a) and 4(c), since the units are the same and the examples shown here are also approximately like $3 \times$ quasi-bulk modulus and $2 \times$ quasi-shear modulus, respectively. 


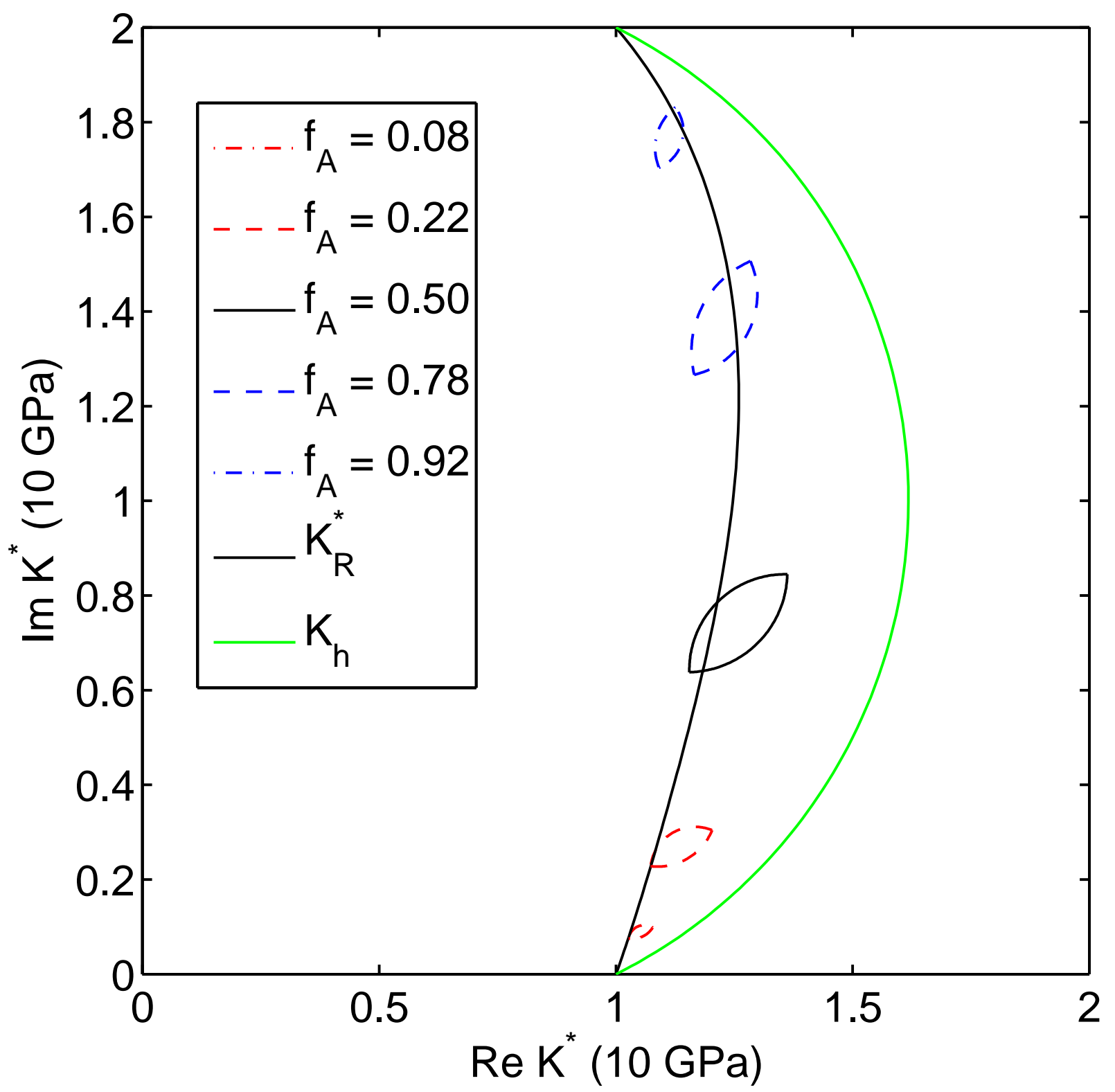

FIG. 8: Illustrating bounds (the closed curves) for the complex bulk modulus $K^{*}$ as the value of the volume fraction $f_{A}$ changes for an overall isotropic binary composite. For comparison, the bulk modulus $K_{R}^{*}$ for the layered case already illustrated in Fig. 5(a) is shown as well, and so is the harmonic mean $K_{h}=\left(f_{A} / K_{A}+f_{B} / K_{B}\right)^{-1}$. We see that $K_{R}^{*}$ tracks the isotropic bounds reasonably well, whereas the harmonic mean is not nearly so close. 